DOI: $10.3901 / J M E .2020 .19 .143$

\title{
广义Waterbomb折纸管的刚性折叠运动特性
}

\author{
冯慧娟 1,2 马家耀 1,2 陈 炎 1,2 \\ (1. 天津大学机构理论与装备设计教育部重点实验室 天津 300350; \\ 2. 天津大学机械工程学院 天津 300350)
}

\begin{abstract}
摘要: 对折叠模式的精确描述是折纸结构工程应用的前提, 但现有的理论和方法无法全面地分析大多数折纸结构的刚性折叠 过程。以广义 Waterbomb 折纸管为研究对象, 基于球面机构运动学理论系统地分析了其折叠行为与运动协调条件, 推导了折 叠管刚性收缩和扭转运动的两套解析运动学方程。研究了各种几何设计参数对 Waterbomb 折纸管刚性折叠行为的影响, 并讨 论了该折纸结构折叠过程中的分岔行为与可能的物理干涉, 及其引起的刚性折叠与结构形变的转化。此工作为基于广义 Waterbomb 折纸管的可编程超材料、可变形结构和机器人的设计与控制奠定了理论基础和设计依据, 同时为复杂折纸结构的 运动学分析提供了有效的手段。
\end{abstract}

关键词: 广义 Waterbomb 折纸管; 刚性折纸; 机构运动学; 机构分岔

中图分类号: TH112

\section{Rigid Folding of Generalized Waterbomb Origami Tubes}

\author{
FENG Huijuan ${ }^{1,2}$ MA Jiayao ${ }^{1,2}$ CHEN Yan $^{1,2}$ \\ (1. Key Laboratory of Mechanism Theory and Equipment Design of Ministry of Education, Tianjin \\ University, Tianjin 300350;
}

2. School of Mechanical Engineering, Tianjin University, Tianjin 300350)

\begin{abstract}
The accurate theoretical description of the folding motion of origami structures is the foundation for their design and precise control in engineering applications. However, the folding behavior of most general origami structures is very difficult to analyze because of the lack of theoretical model and analysis methodology for the complex mobile assemblies of spherical linkages. This paper focuses on the widely-used Waterbomb origami tubes. Based on the kinematics and compatibility of spherical linkages, the rigid folding behavior of generalized Waterbomb tubes was systematically analyzed with analytical kinematics equations to describe their rigid contract and twist motion. The effect of various geometrical parameters on the rigid folding behaviour, bifurcation property as well as physical blockages of the Waterbomb origami tube was studied. This work lays a theoretical foundation for the design and control of programmable metamaterials, deformable structures, and robots based on Waterbomb origami tubes, while such kinematic model can be readily applied to other origami patterns.
\end{abstract}

Key words: Waterbomb origami tube; rigid folding; kinematics; bifurcation

\section{0 前言}

折纸可以通过折叠二维材料形成三维结构, 以 其具有轻质、安全、快速制造、可定制化等优点在 工程领域有着广泛的应用 ${ }^{[1]}$ 。折纸结构的机械性能

* 国家自然科学基金资助项目(51825503, 51721003, 51575377)。20200603 收到初稿, 20200805 收到修改稿
主要取决于其折叠变形模式, 因此折叠模式的精确 理论描述是折纸结构设计与调控的关键。对于给定 的折纸纹路, 目前有多种判断其刚性可折叠性的方 法, 例如 WATANABE 等 ${ }^{[2]}$ 提出的图解和数值方法, $\mathrm{TACHI}$ 的数值算法 ${ }^{[3]}, \mathrm{CAI}$ 等 ${ }^{[4-5]}$ 的四元数旋转序列 与对偶四元数结合的方法, 以及球面机构等效 法 $^{[6-16]}$ 。DAI 等 ${ }^{[6-8]}$ 将折痕和面板分别等效为机构的 关节和连杆, 首次从机构学角度分析了折纸的刚性 折叠。HULL 采用球面三角学分析了一些具有四折 
痕顶点的折纸纹路的刚性可折叠性 ${ }^{[9]}$ 。WU 等 ${ }^{[10]}$ 基 于折纸与球面机构的等效建立了旋转矢量模型, 并 采用四元数和对偶四元数分析了单顶点和多顶点折 纸纹路的刚性可折叠性。STREINU 等 ${ }^{[11]}$ 通过将某些 单顶点折纸等效为球面机构证明了其刚性可折叠 性。 $\mathrm{CHEN}$ 等 ${ }^{[12-14]}$ 通过求解球面机构网格的运动协 调条件给出了柱状折纸的刚性折叠条件。但是目前 已有的分析多集中于一些几何与折叠行为相对简单 的刚性折纸纹路, 而对于具有复杂折叠行为的折纸 结构缺乏有效的理论建模方法。

Waterbomb 折纸管是由六折痕的 Waterbomb 单 元 $^{[17-19]}$ 组成的具有复杂折叠行为的管状折纸结构, 其在工程中有着广泛的应用, 如医用可展折纸支 架 ${ }^{[20]}$ 、可变形机器人车轮 ${ }^{[21-23]}$ 、气动肌肉 ${ }^{[24]}$ 和蠕动 机器人 ${ }^{[25-26]}$ 等。然而, 由于 Waterbomb 折纸管结构 复杂, 对其折叠模式的分析十分困难。作者根据刚 性折纸理论, 将折痕和面板分别等效为机构的关节 和杆件 ${ }^{[6-8]}$, 采用机构运动学理论首次发现了对称假 设条件下的 Waterbomb 折纸管存在刚性扭转运动并 分析了该运动发生的条件及其对结构刚度的影 响 ${ }^{[27]}$ 。同时, 提出了一种基于机构运动学与力学分 析相结合的理论方法解析其径向折叠机理, 获得了 刚度可编程可调控的折纸超材料 ${ }^{[28-29]}$, 且其在运动 过程中具有负泊松比 ${ }^{[30]}$ 。GILLMAN 等 ${ }^{[31]}$ 提出了一 种可用于分析 Waterbomb 折纸管非线性力学问题的 基于桁架的有限元方法。FONSECA 等 ${ }^{[32]}$ 解决了形 状记忆合金驱动的 Waterbomb 可变形机器人车轮的 非线性动力学问题。然而, 在这些分析中, 只讨论 了由矩形 Waterbomb 单元构成的管状折纸结构的折 叠, 而未考虑一般化的非矩形 Waterbomb 单元。 $\mathrm{ZHAO}$ 等 ${ }^{[33-34]}$ 提出了用广义 Waterbomb 单元和一般 六折痕单元构建空间三维曲面的方法。他们仅构建 了复杂的三维曲面, 而未对其折叠行为进行分析。 本文将采用刚性折纸理论分析由广义 Waterbomb 单 元组成的管状折纸结构的刚性折叠行为, 推导其刚性 收缩和扭转运动下的解析运动学方程。研究几何设计 参数对 Waterbomb 折纸管刚性折叠行为与分岔行为 的影响。

\section{1 组成与几何参数}

如图 1a 所示, 广义 Waterbomb 单元包括四条 位于对角线上的谷折痕(虚线)和两条共线的山折痕 (实线), 且所有折痕相交于一个顶点 ${ }^{[17-19]}$, 其中 $a$ 是该单元长度的一半, $\alpha$ 和 $\beta$ 是该单元的设计角
度。将多个这样的 Waterbomb 单元(浅灰)并排组成 一行, 并将相邻行的 Waterbomb 单元(深灰)错开半 个排列, 可形成如图 $1 \mathrm{~b}$ 所示的 Waterbomb 折纸纹 路, 其中, $m$ 和 $n$ 分别是组成该折纸纹路的单元层 数和每层的单元数。将该折纸纹路的两个坚直边粘 连并按折纹折叠, 可获得如图 1c 所示的 Waterbomb 折纸管。由图 $1 \mathrm{~b}$ 可以看出, Waterbomb 折纸管存在 三类不同的六折痕顶点, 分别为图中圆圈标记的 $A_{i}$, $B_{i}$ 和 $C_{i}$, 其中 $i$ 是 Waterbomb 单元所在层的编号。

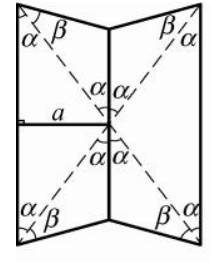

(a) 基本单元

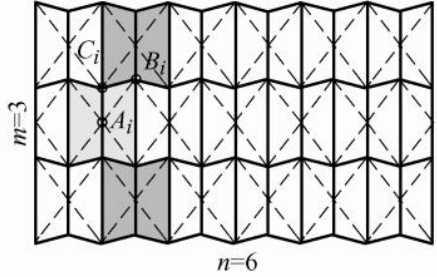

(b) 折纸纹路

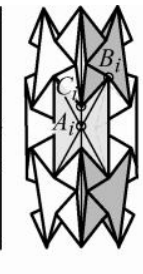

(c) 管状结构

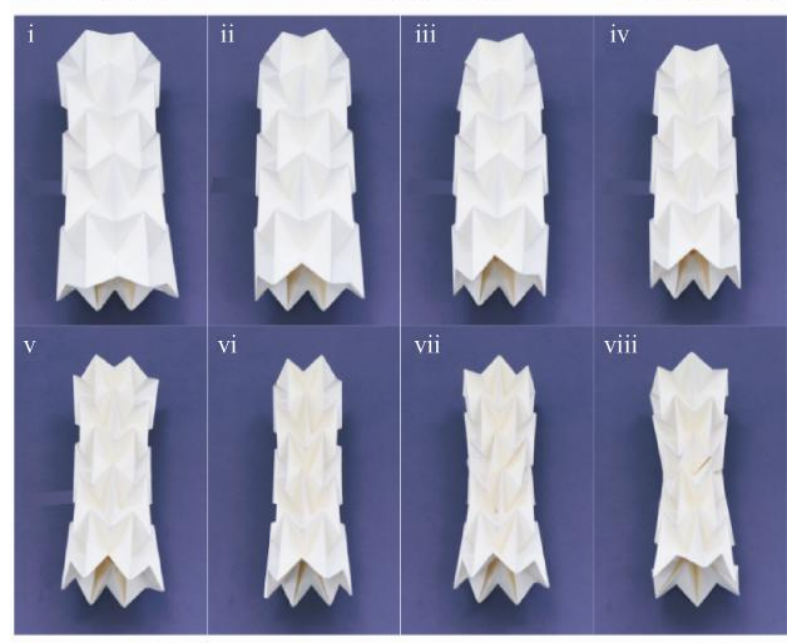

(d) 纸模型折叠过程

图 1 Waterbomb 折纸管的几何设计参数与模型折叠过程

图 1d 给出了一般 Waterbomb 折纸管模型的运 动过程, 其中纸模型的设计参数为 $m=5, n=6$, $\alpha=\beta=40^{\circ}$ 。该管子运动从未端层完全展开的构型 $\mathrm{i}$ 开始, 随着管子的收缩, 其直径和长度均减小, 管 子到达等直径构型 ii。当管子继续收缩, 经过构型 iii，其再次达到等直径构型 iv。当中间层完全收缩 时(构型 vi), 该管子到达径向收缩运动的极限。此 时，若进一步给管子一个小扰动，该管子将从中间 层开始发生扭转运动(构型 vii)。当组成中间层的广 义 Waterbomb 单元中大三角形和小三角形重合时 (构型 viii)，该管子到达扭转运动的极限。

\section{2 径向收缩运动}

在刚性折纸的分析中, 可将折痕和面板分别视 为机构的关节和连杆, 则每个单顶点可等效为一个 
球面机构 $^{[6-8]}$, 且多个单顶点组成的折纸纹路可视为 多个球面机构构成的网格 ${ }^{[35-36]}$ 。因此, Waterbomb 折纸管中的三个典型顶点 $A_{i}, B_{i}$ 和 $C_{i}$ 可以看作是三 种球面六杆机构, 如图 2 所示, 各顶点处相邻面板 间的折叠二面角分别为 $\eta_{i, j}, \kappa_{B i, j}$ 和 $\kappa_{C i, j}(j=1,2, \cdots, 6)$ 。

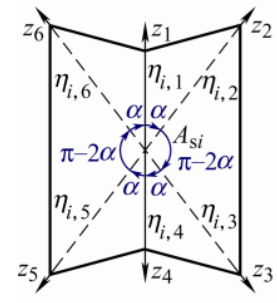

(a) 球面机构 $A_{\mathrm{s} i}$

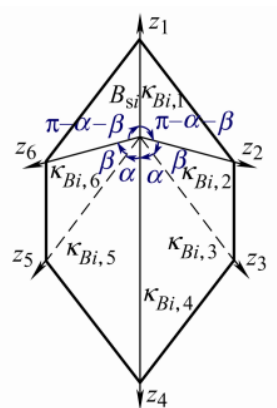

(b) 球面机构 $B_{\mathrm{s} i}$

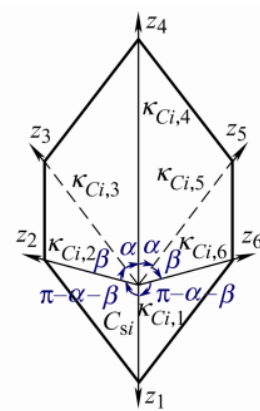

(c) 球面机构 $C_{\mathrm{s} i}$
图 2 Waterbomb 折纸管的等效运动学模型

在一般情况下, 单个球面六杆机构的自由度为 3, 由此组成的 Waterbomb 折纸管是个多自由度系 统。此处, 我们主要分析在轴对称情况下该折纸管 的刚性折叠行为。通过观察图 1d 中广义 Waterbomb 折纸管模型的折叠运动, 我们做出以下假设: (1) 位 于同一层的所有单元运动同步; (2) 在运动过程中, 每个基本单元的运动都是面对称或中心对称; (3) 该 折纸管由一个赤道面平分为上下两部分, 且两部分 的运动对称。

根据 Denavit-Hartenberg(D-H) 法 ${ }^{[37-38]}$ 建立坐标 系, 如图 3 所示, $z_{k}$ 轴沿着关节 $k$ 方向, $x_{k}$ 轴沿着 $z_{k-1}$ 轴和 $z_{k}$ 轴的法线方向且由 $z_{k-1}$ 指向 $z_{k}, y_{k}$ 轴由右手 定则确定。几何参数 $\alpha_{k(k+1)}$ 为 $z_{k}$ 轴与 $z_{k+1}$ 轴间的夹 角, 正方向与 $x_{k+1}$ 轴一致。运动变量 $\theta_{k}$ 为关节 $k$ 处 的转动角, 即 $x_{k}$ 轴到 $x_{k+1}$ 轴的夹角, 正方向与 $z_{k}$ 轴 一致。由此可建立球面六杆机构的闭环方程, 即

$$
\boldsymbol{Q}_{21} \boldsymbol{Q}_{32} \boldsymbol{Q}_{43} \boldsymbol{Q}_{54} \boldsymbol{Q}_{65} \boldsymbol{Q}_{16}=\boldsymbol{I}_{3}
$$

其中, 变换矩阵 $\boldsymbol{Q}_{(k+1) k}$ 为

$$
\boldsymbol{Q}_{(k+1) k}=\left[\begin{array}{ccc}
\mathrm{c} \theta_{k} & -\mathrm{c} \alpha_{k(k+1)} \mathrm{s} \theta_{k} & \mathrm{~s} \alpha_{k(k+1)} \mathrm{s} \theta_{k} \\
\mathrm{~s} \theta_{k} & \mathrm{c} \alpha_{k(k+1)} \mathrm{c} \theta_{k} & -\mathrm{s} \alpha_{k(k+1)} \mathrm{c} \theta_{k} \\
0 & \mathrm{~s} \alpha_{k(k+1)} & \mathrm{c} \alpha_{k(k+1)}
\end{array}\right]
$$

式中, $\mathrm{s}$ 和 $\mathrm{c}$ 分别为正弦函数 $\sin$ 和余弦函数 $\cos$ 的 简写, 后文亦同。该变换矩阵可将第 $k+1$ 个坐标系 中的表达转换到第 $k$ 个坐标系中, 且当 $k+1>6$, 取 $k+1$ 为 1 。

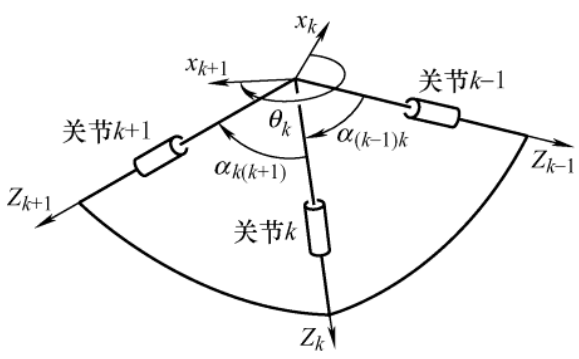

图 3 球面机构的 D-H 建系法及参数

如图 2a 所示, 球面六杆机构 $A_{\mathrm{s} i}$ 的几何参数为 $\alpha_{23}^{A}=\alpha_{56}^{A}=\pi-2 \alpha, \quad \alpha_{12}^{A}=\alpha_{34}^{A}=\alpha_{45}^{A}=\alpha_{61}^{A}=\alpha$ 。根据 面对称条件, 该机构的运动变量 $\delta_{i, j}(j=1,2, \ldots, 6)$ 存 在如下关系： $\delta_{i, 5}=\delta_{i, 3}, \quad \delta_{i, 6}=\delta_{i, 2}$ 。将其代入式(1) 中, 可得

$\tan \frac{\delta_{i, 1}}{2}=\frac{\left[\begin{array}{l}-\mathrm{s} \alpha\left(\mathrm{s} \alpha \mathrm{s} 2 \alpha \mathrm{c} \delta_{i, 3}+\mathrm{c} \alpha \mathrm{c} 2 \alpha \mathrm{c} \delta_{i, 2} \mathrm{c} \delta_{i, 3}+\right. \\ \left.\mathrm{c} \alpha \mathrm{s} \delta_{i, 2} \mathrm{~s} \delta_{i, 3}\right)+\mathrm{c} \alpha\left(\mathrm{c} \alpha \mathrm{s} 2 \alpha \mathrm{c} \delta_{i, 2}-\mathrm{s} \alpha \mathrm{c} 2 \alpha\right)\end{array}\right]}{\mathrm{s} \alpha\left(\mathrm{s} \delta_{i, 3} \mathrm{c} \delta_{i, 2}-\mathrm{c} 2 \alpha \mathrm{s} \delta_{i, 2} \mathrm{c} \delta_{i, 3}\right)+\mathrm{c} \alpha \mathrm{s} 2 \alpha \mathrm{s} \delta_{i, 2}}$

$\tan \frac{\delta_{i, 4}}{2}=\frac{\left[\begin{array}{l}\mathrm{c} \alpha\left(\mathrm{c} \alpha \mathrm{s} 2 \alpha \mathrm{c} \delta_{i, 3}-\mathrm{s} \alpha \mathrm{c} 2 \alpha \mathrm{c} \delta_{i, 2} \mathrm{c} \delta_{i, 3}-\right. \\ \left.\mathrm{s} \alpha \mathrm{s} \delta_{i, 2} \mathrm{~s} \delta_{i, 3}\right)-\mathrm{s} \alpha\left(\mathrm{s} \alpha \mathrm{s} 2 \alpha \mathrm{c} \delta_{i, 2}+\mathrm{c} \alpha \mathrm{c} 2 \alpha\right)\end{array}\right]}{\mathrm{s} \alpha\left(\mathrm{s} \delta_{i, 2} \mathrm{c} \delta_{i, 3}-\mathrm{c} 2 \alpha \mathrm{s} \delta_{i, 3} \mathrm{c} \delta_{i, 2}\right)+\mathrm{c} \alpha \mathrm{s} 2 \alpha \mathrm{s} \delta_{i, 3}}$

将运动变量 $\delta_{i, j}$ 和二面角 $\eta_{i, j}$ 间的关系代入公式(3), 即 $\delta_{i, 1}=\pi-\eta_{i, 1} ， \delta_{i, 2}=\pi+\eta_{i, 2} ， \delta_{i, 3}=\pi+\eta_{i, 3}$ ， $\delta_{i, 4}=\pi-\eta_{i, 4}, \quad \delta_{i, 5}=\pi+\eta_{i, 5}, \quad \delta_{i, 6}=\pi+\eta_{i, 6}$, 可得 $\tan \frac{\eta_{i, 1}}{2}=\frac{\mathrm{s} \alpha\left(\mathrm{s} \eta_{i, 3} \mathrm{c} \eta_{i, 2}-\mathrm{c} 2 \alpha \mathrm{s} \eta_{i, 2} \mathrm{c} \eta_{i, 3}\right)-\mathrm{c} \alpha \mathrm{s} 2 \alpha \mathrm{s} \eta_{i, 2}}{\left[\begin{array}{l}\mathrm{s} \alpha\left(\mathrm{s} \alpha \mathrm{s} 2 \alpha \mathrm{c} \eta_{i, 3}-\mathrm{c} \alpha \mathrm{c} 2 \alpha \mathrm{c} \eta_{i, 2} \mathrm{c} \eta_{i, 3}-\right. \\ \left.\mathrm{c} \alpha \mathrm{s} \eta_{i, 2} \mathrm{~s} \eta_{i, 3}\right)-\mathrm{c} \alpha\left(\mathrm{c} \alpha \mathrm{s} 2 \alpha \mathrm{c} \eta_{i, 2}+\mathrm{s} \alpha \mathrm{c} 2 \alpha\right)\end{array}\right]}$

$\tan \frac{\eta_{i, 4}}{2}=\frac{\mathrm{s} \alpha\left(\mathrm{s} \eta_{i, 2} \mathrm{c} \eta_{i, 3}-\mathrm{c} 2 \alpha \mathrm{s} \eta_{i, 3} \mathrm{c} \eta_{i, 2}\right)-\mathrm{c} \alpha \mathrm{s} 2 \alpha \mathrm{s} \eta_{i, 3}}{\left[\begin{array}{l}-\mathrm{c} \alpha\left(\mathrm{c} \alpha \mathrm{s} 2 \alpha \mathrm{c} \eta_{i, 3}+\mathrm{s} \alpha \mathrm{c} 2 \alpha \mathrm{c} \eta_{i, 2} \mathrm{c} \eta_{i, 3}+\right. \\ \left.\mathrm{s} \alpha \mathrm{s} \eta_{i, 2} \mathrm{~s} \eta_{i, 3}\right)+\mathrm{s} \alpha\left(\mathrm{s} \alpha \mathrm{s} 2 \alpha \mathrm{c} \eta_{i, 2}-\mathrm{c} \alpha \mathrm{c} 2 \alpha\right)\end{array}\right]}$

$$
\eta_{i, 5}=\eta_{i, 3} \quad \eta_{i, 6}=\eta_{i, 2}
$$

如图 $2 \mathrm{~b}$ 所示, 面对称球面六杆机构 $B_{\mathrm{si}}$ 的几何 参数为 $\alpha_{12}^{B}=\alpha_{61}^{B}=\pi-\alpha-\beta, \alpha_{23}^{B}=\alpha_{56}^{B}=\beta$, $\alpha_{34}^{B}=\alpha_{45}^{B}=\alpha$, 且其运动变量 $\omega_{B i, j}(j=1,2, \cdots, 6)$ 有 以下关系

$$
\omega_{B i, 5}=\omega_{B i, 3} \quad \omega_{B i, 6}=\omega_{B i, 2}
$$

将式(5a)代入式(1), 可得 
$\tan \frac{\omega_{B i, 1}}{2}=\frac{\left(\begin{array}{l}\mathrm{s} \alpha\left[-\mathrm{c}(\alpha+\beta) \mathrm{c} \beta \mathrm{c} \omega_{B i, 2} \mathrm{c} \omega_{B i, 3}+\right. \\ \left.\mathrm{c}(\alpha+\beta) \mathrm{s} \omega_{B i, 2} \mathrm{~s} \omega_{B i, 3}-\mathrm{s}(\alpha+\beta) \mathrm{s} \beta \mathrm{c} \omega_{B i, 3}\right]+ \\ \mathrm{c} \alpha\left[\begin{array}{l}\left.\mathrm{s}(\alpha+\beta) \mathrm{c} \beta-\mathrm{c}(\alpha+\beta) \mathrm{s} \beta \mathrm{c} \omega_{B i, 2}\right]\end{array}\right.\end{array}\right)}{\left[\begin{array}{l}\mathrm{s} \alpha\left(\mathrm{s} \omega_{B i, 3} \mathrm{c} \omega_{B i, 2}+\mathrm{c} \beta \mathrm{s} \omega_{B i, 2} \mathrm{c} \omega_{B i, 3}\right)+ \\ \mathrm{c} \alpha \mathrm{s} \beta \mathrm{s} \omega_{B i, 2}\end{array}\right]}$

$\tan \frac{\omega_{B i, 4}}{2}=\frac{\left(\begin{array}{l}\mathrm{c} \alpha\left[\mathrm{s}(\alpha+\beta)\left(\mathrm{c} \beta \mathrm{c} \omega_{B i, 2} \mathrm{c} \omega_{B i, 3}-\mathrm{s} \omega_{B i, 2} \mathrm{~s} \omega_{B i, 3}\right)-\right. \\ \left.\mathrm{c}(\alpha+\beta) \mathrm{s} \beta \mathrm{c} \omega_{B i, 3}\right]-\mathrm{s} \alpha[\alpha+\beta) \mathrm{c} \beta+ \\ \left.\mathrm{s}(\alpha+\beta) \mathrm{s} \beta \mathrm{c} \omega_{B i, 2}\right]\end{array}\right.}{\left[\begin{array}{l}\mathrm{s}(\alpha+\beta)\left(\mathrm{s} \omega_{B i, 2} \mathrm{c} \omega_{B i, 3}+\mathrm{c} \beta \mathrm{s} \omega_{B i, 3} \mathrm{c} \omega_{B i, 2}\right)- \\ \mathrm{c}(\alpha+\beta) \mathrm{s} \beta \mathrm{s} \omega_{B i, 3}\end{array}\right]}$

对于球面六杆机构 $B_{\mathrm{s} i}$, 其运动变量 $\omega_{B i, j}$ 和二面角 $\kappa_{B i, j}$ 间的关系为 $\omega_{B i, 1}=\pi-\kappa_{B i, 1}, \omega_{B i, 2}=\pi-\kappa_{B i, 2}$, $\omega_{B i, 3}=\pi+\kappa_{B i, 3}, \quad \omega_{B i, 4}=\pi-\kappa_{B i, 4}, \quad \omega_{B i, 5}=\pi+\kappa_{B i, 5}$, $\omega_{B i, 6}=\pi-\kappa_{B i, 6}$ 。将此关系代入式(5)中, 可得球面机 构 $B_{\mathrm{s} i}$ 中各二面角间的运动关系为

$$
\kappa_{B i, 5}=\kappa_{B i, 3} \quad \kappa_{B i, 6}=\kappa_{B i, 2}
$$

$\tan \frac{\kappa_{B i, 1}}{2}=\frac{\left[\begin{array}{l}\mathrm{s} \alpha\left(\mathrm{s} \kappa_{B i, 3} \mathrm{c} \kappa_{B i, 2}-\mathrm{c} \beta \mathrm{s} \kappa_{B i, 2} \mathrm{c} \kappa_{B i, 3}\right)+ \\ \mathrm{c} \alpha \mathrm{s} \beta \mathrm{s} \kappa_{B i, 2}\end{array}\right]}{\left(\begin{array}{l}\mathrm{s} \alpha\left[\begin{array}{l}\mathrm{s}(\alpha+\beta) \mathrm{s} \beta \mathrm{c} \kappa_{B i, 3}-\mathrm{c}(\alpha+\beta) \mathrm{s} \kappa_{B i, 2} \mathrm{~s} \kappa_{B i, 3}- \\ \left.\mathrm{c}(\alpha+\beta) \mathrm{c} \beta \mathrm{c} \kappa_{B i, 2} \mathrm{c} \kappa_{B i, 3}\right]+ \\ \mathrm{c} \alpha\left[\mathrm{s}(\alpha+\beta) \mathrm{c} \beta+\mathrm{c}(\alpha+\beta) \mathrm{s} \beta \mathrm{c} \kappa_{B i, 2}\right]\end{array}\right.\end{array}\right)}$

$$
\tan \frac{\kappa_{B i, 4}}{2}=\frac{\left[\begin{array}{l}
\mathrm{s}(\alpha+\beta)\left(\mathrm{c} \beta \mathrm{s} \kappa_{B i, 3} \mathrm{c} \kappa_{B i, 2}-\mathrm{s} \kappa_{B i, 2} \mathrm{c} \kappa_{B i, 3}\right)+ \\
\mathrm{c}(\alpha+\beta) \mathrm{s} \beta \mathrm{s} \kappa_{B i, 3}
\end{array}\right]}{\left(\begin{array}{l}
\mathrm{c} \alpha\left[\mathrm{s}(\alpha+\beta) \mathrm{c} \beta \mathrm{c} \kappa_{B i, 2} \mathrm{c} \kappa_{B i, 3}+\right. \\
\left.\mathrm{s}(\alpha+\beta) \mathrm{s} \kappa_{B i, 2} \mathrm{~s} \kappa_{B i, 3}+\mathrm{c}(\alpha+\beta) \mathrm{s} \beta \mathrm{c} \kappa_{B i, 3}\right]+ \\
\mathrm{s} \alpha\left[\mathrm{s}(\alpha+\beta) \mathrm{s} \beta \mathrm{c} \kappa_{B i, 2}-\mathrm{c}(\alpha+\beta) \mathrm{c} \beta\right]
\end{array}\right.}
$$

同理, 图 2c 所示的面对称球面六杆机构 $C_{\mathrm{s} i}$ 中 各二面角间的运动关系为

$$
\begin{gathered}
\kappa_{C i, 5}=\kappa_{C i, 3} \quad \kappa_{C i, 6}=\kappa_{C i, 2} \\
\tan \frac{\kappa_{C i, 1}}{2}=\frac{\left[\begin{array}{l}
\mathrm{s} \alpha\left(\mathrm{s} \kappa_{C i, 3} \mathrm{c} \kappa_{C i, 2}-\mathrm{c} \beta \mathrm{s} \kappa_{C i, 2} \mathrm{c} \kappa_{C i, 3}\right)+ \\
\mathrm{c} \alpha \mathrm{s} \beta \mathrm{s} \kappa_{C i, 2}
\end{array}\right]}{\left(\begin{array}{l}
\mathrm{s} \alpha\left[\mathrm{s}(\alpha+\beta) \mathrm{s} \beta \mathrm{c} \kappa_{C i, 3}-\mathrm{c}(\alpha+\beta) \mathrm{s} \kappa_{C i, 2} \mathrm{~s} \kappa_{C i, 3}-\right. \\
\left.\mathrm{c}(\alpha+\beta) \mathrm{c} \beta \mathrm{c} \kappa_{C i, 2} \mathrm{c} \kappa_{C i, 3}\right]+ \\
\mathrm{c} \alpha\left[\mathrm{s}(\alpha+\beta) \mathrm{c} \beta+\mathrm{c}(\alpha+\beta) \mathrm{s} \beta \mathrm{c} \kappa_{C i, 2}\right]
\end{array}\right)}
\end{gathered}
$$

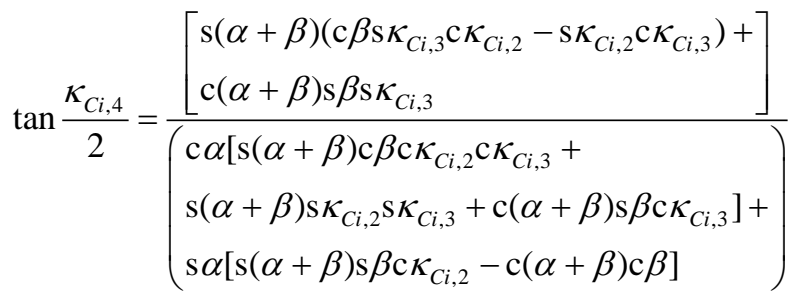

在图 1c 所示的折纸纹路中, 每个折痕连接两个 顶点，则该折痕上的二面角与两顶点处的球面机构 的运动均相关, 故相邻球面机构 $A_{\mathrm{s} i}, B_{\mathrm{s} i}, C_{\mathrm{s} i}$ 间的 运动协调条件为

$$
\begin{gathered}
\kappa_{B i, 3}=\eta_{i, 6} \quad \kappa_{C i, 1}=\eta_{i, 1} \quad \kappa_{C i, 2}=\kappa_{B i, 2} \\
\eta_{i+1,4}=\kappa_{B i, 1} \quad \eta_{i+1,3}=\kappa_{C i, 3}
\end{gathered}
$$

式(8)适用于整个 Waterbomb 折纸管。由式(4)、(6) (8) 可知, 此时该结构仅满足运动假设条件(1)、(2)，仍需 3 个变量才能确定其运动, 即其自由度为 3 。下面我们将 考虑运动假设条件(3)及管状结构的周向闭环条件以 进一步确定 Waterbomb 折纸管的运动。由于该折纸管 的运动假设条件(3)受其单元层数 $m$ 的影响, 此处我们 将其分为 $m$ 为奇数和 $m$ 为偶数两种情况讨论。

\section{$2.1 \mathrm{~m}$ 为奇数}

此种情况下，Waterbomb 折纸管存在一个中间 层, 我们将其定义为 0 层。考虑运动假设条件(3)中 的纵向对称性, 球面六杆机构 $A_{\mathrm{s} 0}$ 的运动不仅满足 面对称条件, 还需满足线对称条件, 即该机构的上 下两部分关于过顶点 $A_{0}$ 且垂直于折纸管的中心轴 旋转对称。故球面机构 $A_{\mathrm{s} 0}$ 的二面角满足以下关系 $\tan \frac{\eta_{0,2}}{2}=\operatorname{c} \alpha \tan \frac{\eta_{0,1}}{2} \quad \eta_{0,4}=\eta_{0,1} \quad \eta_{0,2}=\eta_{0,3}=\eta_{0,5}=\eta_{0,6}$

Waterbomb 折纸管的三维图及 0 层在赤道面上 的投影如图 4 所示, 其中 $B_{0} P_{0} \perp A_{0} P_{0}, B_{0}^{\prime} P_{0} \perp A_{0} P_{0}$, $P_{0}$ 为垂足。 $E$ 和 $E^{\prime}$ 为 0 层单元坚直边的中点。由于 $\overline{E E^{\prime}}=\overline{B_{0} B_{0}^{\prime}}, \overline{E E^{\prime}}=2 a \mathrm{~s} \frac{\theta}{2}, \overline{B_{0} B_{0}^{\prime}}=2 a \mathrm{~s} \frac{\eta_{0,1}}{2}$, 可知

$$
\theta=\eta_{0,1}
$$

式中, $\theta$ 为以 $A_{0}$ 为顶点的单元中两个最大三角形面 板间的二面角, 如图 $4 \mathrm{a}$ 所示。由图 $4 \mathrm{~b}$ 可以看出, 该折纸管的周向闭环条件为

$$
\frac{\kappa_{B 0,4}}{2}+\frac{180^{\circ}}{n}=\frac{\eta_{0,1}}{2}
$$

因此，当 $m$ 为奇数时, Waterbomb 折纸管中各 二面角间的运动关系如下。对于球面机构 $A_{\mathrm{s} 0}$ $\tan \frac{\eta_{0,2}}{2}=\operatorname{c} \alpha \tan \frac{\eta_{0,1}}{2} \quad \eta_{0,4}=\eta_{0,1} \quad \eta_{0,2}=\eta_{0,3}=\eta_{0,5}=\eta_{0,6}$ 


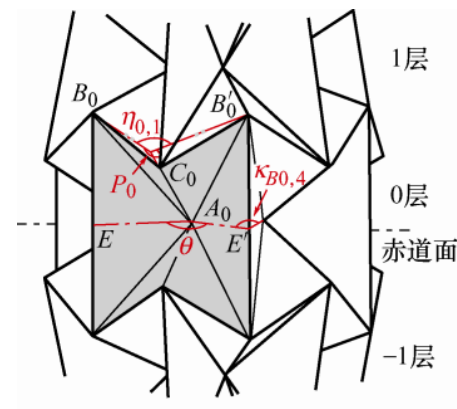

(a) 三维图

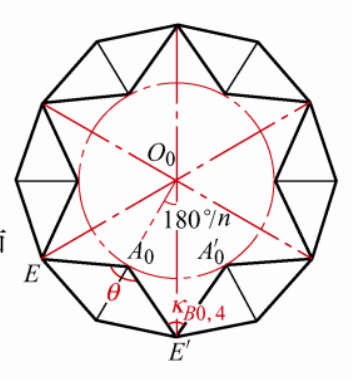

(b) 0层在赤道面的投影

图 $4 m$ 为奇数的 Waterbomb 折纸管

对于球面机构 $B_{\mathrm{s} 0}$

$$
\begin{aligned}
& \kappa_{B 0,4}=\eta_{0,1}-\frac{2 \pi}{n} \kappa_{B 0,3}=\kappa_{B 0,5}=\eta_{0,6} \quad \kappa_{B 0,6}=\kappa_{B 0,2} \\
& \tan \frac{\kappa_{B 0,2}}{2}=\frac{\left(\begin{array}{l}
\mathrm{s}(\alpha+\beta)\left(\mathrm{c} \alpha \tan \frac{\kappa_{B 0,4}}{2} \mathrm{~s} \kappa_{B 0,3}+\mathrm{c} \kappa_{B 0,3}\right)- \\
\left\{\mathrm{s}^{2}(\alpha+\beta)\left(\mathrm{c} \alpha \tan \frac{\kappa_{B 0,4}}{2} \mathrm{~s} \kappa_{B 0,3}+\mathrm{c} \kappa_{B 0,3}\right)^{2}+\right. \\
\mathrm{s} \alpha\left[\mathrm{c} \alpha \mathrm{s}(\alpha+2 \beta) \tan \frac{\kappa_{B 0,4}}{2} \mathrm{c} \kappa_{B 0,3}-\right. \\
\left.\mathrm{s} \alpha \mathrm{c}(\alpha+2 \beta) \tan \frac{\kappa_{B 0,4}}{2}-\mathrm{s}(\alpha+2 \beta) \mathrm{s} \kappa_{B 0,3}\right] \\
\left.\left[\mathrm{c} \alpha \tan \frac{\kappa_{B 0,4}}{2}\left(\mathrm{c} \kappa_{B 0,3}+1\right)-\mathrm{s} \kappa_{B 0,3}\right]\right\}^{1 / 2}
\end{array}\right.}{\mathrm{s} \alpha\left[\mathrm{c} \alpha \tan \frac{\kappa_{B 0,4}}{2}\left(\mathrm{c} \kappa_{B 0,3}+1\right)-\mathrm{s} \kappa_{B 0,3}\right]} \\
& \tan \frac{\kappa_{B 0,1}}{2}=\frac{\left[\begin{array}{l}
\mathrm{s} \alpha\left(\mathrm{s} \kappa_{B 0,3} \mathrm{c} \kappa_{B 0,2}-\mathrm{c} \beta \mathrm{s} \kappa_{B 0,2} \mathrm{c} \kappa_{B 0,3}\right)+ \\
\mathrm{c} \alpha \mathrm{s} \beta \mathrm{s} \kappa_{\mathrm{B} 0,2}
\end{array}\right]}{\begin{array}{l}
\mathrm{s} \alpha\left[\mathrm{s}(\alpha+\beta) \mathrm{s} \beta \mathrm{c} \kappa_{B 0,3}-\mathrm{c}(\alpha+\beta) \mathrm{s} \kappa_{B 0,2} \mathrm{~s} \kappa_{B 0,3}-\right. \\
\left.\mathrm{c}(\alpha+\beta) \mathrm{c} \beta \mathrm{c} \kappa_{B 0,2} \mathrm{c} \kappa_{B 0,3}\right]+\mathrm{c} \alpha[\mathrm{s}(\alpha+\beta) \mathrm{c} \beta+ \\
\left.\mathrm{c}(\alpha+\beta) \mathrm{s} \beta \mathrm{c} \kappa_{B 0,2}\right]
\end{array}}
\end{aligned}
$$

对于球面机构 $C_{\mathrm{s} 0}$

$$
\begin{gathered}
\kappa_{C 0,1}=\eta_{0,1} \quad \kappa_{C 0,2}=\kappa_{C 0,6}=\kappa_{B 0,2} \quad \kappa_{C 0,5}=\kappa_{C 0,3} \\
\tan \frac{\kappa_{C 0,3}}{2}=\frac{\left(\begin{array}{l}
\mathrm{s} \alpha \mathrm{c}(\alpha+\beta) \tan \frac{\kappa_{C 0,1}}{2} \mathrm{~s} \kappa_{C 0,2}+\mathrm{s} \alpha \mathrm{c} \kappa_{C 0,2}- \\
\left\{\left[\mathrm{s} \alpha \mathrm{c} \kappa_{C 0,2}+\mathrm{s} \alpha \mathrm{c}(\alpha+\beta) \tan \frac{\kappa_{C 0,1}}{2} \mathrm{~s} \kappa_{C 0,2}\right]^{2}-\right. \\
\mathrm{s}(\alpha+\beta)\left[\mathrm{c}(\alpha+\beta) \mathrm{s}(\beta-\alpha) \tan \frac{\kappa_{C 0,1}}{2} \mathrm{c} \kappa_{C 0,2}-\right. \\
\left.\mathrm{s}(\beta-\alpha) \mathrm{s} \kappa_{C 0,2}+\mathrm{s}(\alpha+\beta) \mathrm{c}(\beta-\alpha) \tan \frac{\kappa_{C 0,1}}{2}\right] \\
\left.\left[\mathrm{c}(\alpha+\beta) \tan \frac{\kappa_{C 0,1}}{2}\left(\mathrm{c} \kappa_{C 0,2}+1\right)-\mathrm{s} \kappa_{C 0,2}\right]\right\}^{1 / 2}
\end{array}\right)}{\mathrm{s}(\alpha+\beta)\left[\mathrm{c}(\alpha+\beta) \tan \frac{\kappa_{C 0,1}}{2}\left(\mathrm{c} \kappa_{C 0,2}+1\right)-\mathrm{s} \kappa_{C 0,2}\right]}
\end{gathered}
$$

$$
\tan \frac{\kappa_{C 0,4}}{2}=\frac{\left[\begin{array}{l}
\mathrm{s}(\alpha+\beta)\left(\mathrm{c} \beta \mathrm{c} \kappa_{C 0,2} \mathrm{~s} \kappa_{C 0,3}-\mathrm{s} \kappa_{C 0,2} \mathrm{c} \kappa_{C 0,3}\right)+ \\
\mathrm{c}(\alpha+\beta) \mathrm{s} \beta \mathrm{s} \kappa_{C 0,3}
\end{array}\right]}{\left(\begin{array}{l}
\mathrm{c} \alpha\left[\mathrm{s}(\alpha+\beta) \mathrm{c} \beta \mathrm{c} \kappa_{C 0,2} \mathrm{c} \kappa_{C 0,3}+\right. \\
\left.\mathrm{c}(\alpha+\beta) \mathrm{s} \beta \mathrm{c} \kappa_{C 0,3}+\mathrm{s}(\alpha+\beta) \mathrm{s} \kappa_{C 0,2} \mathrm{~s} \kappa_{C 0,3}\right]+ \\
\mathrm{s} \alpha\left[\mathrm{s}(\alpha+\beta) \mathrm{s} \beta \mathrm{c} \kappa_{C 0,2}-\mathrm{c}(\alpha+\beta) \mathrm{c} \beta\right]
\end{array}\right)}
$$

对于球面机构 $A_{\mathrm{s} 1}$

$$
\begin{gathered}
\eta_{1,3}=\eta_{1,5}=\kappa_{C 0,3} \eta_{1,4}=\kappa_{B 0,1} \quad \eta_{1,2}=\eta_{1,6} \\
\tan \frac{\eta_{1,6}}{2}=\frac{\left(\begin{array}{l}
\mathrm{c} \eta_{1,5}+\tan \frac{\eta_{1,4}}{2} \mathrm{c} \alpha \mathrm{s} \eta_{1,5}-\left\{\left(\tan \frac{\eta_{1,4}}{2} \mathrm{c} \alpha \mathrm{s} \eta_{1,5}+\right.\right. \\
\left.\mathrm{c} \eta_{1,5}\right)^{2}-\left(2 \mathrm{c}^{2} \alpha+\mathrm{c} 2 \alpha\right)\left[\mathrm{s} \eta_{1,5}-\right. \\
\left.\tan \frac{\eta_{1,4}}{2} \mathrm{c} \alpha\left(\mathrm{c} \eta_{1,5}+1\right)\right]^{2}-2 \mathrm{c} \alpha \tan \frac{\eta_{1,4}}{2}\left[\mathrm{~s} \eta_{1,5}-\right. \\
\left.\left.\tan \frac{\eta_{1,4}}{2} \mathrm{c} \alpha\left(\mathrm{c} \eta_{1,5}+1\right)\right]\right\}^{1 / 2}
\end{array}\right)}{\tan \frac{\eta_{1,1}}{2}=\frac{\operatorname{s} \eta_{1,5}-\tan \frac{\eta_{1,4}}{2} \mathrm{c} \alpha\left(\mathrm{s} \eta_{1,5} \mathrm{c} \eta_{1,6}-\mathrm{c} 2 \alpha \mathrm{s} \eta_{1,5} \mathrm{c} \eta_{1,5}\right)-\mathrm{c} \alpha \mathrm{s} 2 \alpha \mathrm{s} \eta_{1,6}}{\left(\begin{array}{l}
-\mathrm{c} \alpha\left(\mathrm{c} \alpha \mathrm{s} 2 \alpha \mathrm{c} \eta_{1,6}+\mathrm{s} \alpha \mathrm{c} 2 \alpha \mathrm{c} \eta_{1,5} \mathrm{c} \eta_{1,6}+\right. \\
\left.\mathrm{s} \alpha \mathrm{s} \eta_{1,5} \mathrm{~s} \eta_{1,6}\right)+\mathrm{s} \alpha\left(\mathrm{s} \alpha \mathrm{s} 2 \alpha \mathrm{c} \eta_{1,5}-\mathrm{c} \alpha \mathrm{c} 2 \alpha\right)
\end{array}\right)}}
\end{gathered}
$$

对于球面机构 $B_{\mathrm{s} i}$

$$
\begin{aligned}
& \kappa_{B i, 3}=\kappa_{B i, 5}=\eta_{i, 6} \quad \kappa_{B i, 4}=\kappa_{C(i-1), 4} \quad \kappa_{B i, 6}=\kappa_{B i, 2} \\
& \left(\mathrm{~s}(\alpha+\beta)\left(\mathrm{c} \alpha \tan \frac{\kappa_{B i, 4}}{2} \mathrm{~s} \kappa_{B i, 3}+\mathrm{c} \kappa_{B i, 3}\right)-\right. \\
& \left\{\mathrm { s } ^ { 2 } ( \alpha + \beta ) \left(\mathrm{c} \alpha \tan \frac{\kappa_{B i, 4}}{2} \mathrm{~s} \kappa_{B i, 3}+\right.\right. \\
& \left.\mathrm{c} \kappa_{B i, 3}\right)^{2}+\mathrm{s} \alpha\left[\mathrm{c} \alpha \tan \frac{\kappa_{B i, 4}}{2}\left(\mathrm{c} \kappa_{B i, 3}+1\right)-\right. \\
& \left.\mathrm{s} \kappa_{B i, 3}\right]\left[\mathrm{c} \alpha S(\alpha+2 \beta) \tan \frac{\kappa_{B i, 4}}{2} \mathrm{c} \kappa_{B i, 3}-\right. \\
& \tan \frac{\kappa_{B i, 2}}{2}=\frac{\left.\left.\left(\mathrm{s}(\alpha+2 \beta) \mathrm{s} \kappa_{B i, 3}-\mathrm{s} \alpha \mathrm{c}(\alpha+2 \beta) \tan \frac{\kappa_{B i, 4}}{2}\right]\right\}^{1 / 2}\right)}{\mathrm{s} \alpha\left[\mathrm{c} \alpha \tan \frac{\kappa_{B i, 4}}{2}\left(\mathrm{c} \kappa_{B i, 3}+1\right)-\mathrm{s} \kappa_{B i, 3}\right]} \\
& \tan \frac{\kappa_{B i, 1}}{2}=\frac{\left[\begin{array}{l}
\mathrm{s} \alpha\left(\mathrm{s} \kappa_{B i, 3} \mathrm{c} \kappa_{B i, 2}-\mathrm{c} \beta \mathrm{s} \kappa_{B i, 2} \mathrm{c} \kappa_{B i, 3}\right)+ \\
\mathrm{c} \alpha \mathrm{s} \beta \mathrm{s} \kappa_{B i, 2}
\end{array}\right]}{\left(\begin{array}{l}
\mathrm{s} \alpha\left[-\mathrm{c}(\alpha+\beta) \mathrm{c} \beta \mathrm{c} \kappa_{B i, 2} \mathrm{c} \kappa_{B i, 3}-\right. \\
\left.\mathrm{c}(\alpha+\beta) \mathrm{s} \kappa_{B i, 2} \mathrm{~s} \kappa_{B i, 3}+\mathrm{s}(\alpha+\beta) \mathrm{s} \beta \mathrm{c} \kappa_{B i, 3}\right]+ \\
\mathrm{c} \alpha\left[\mathrm{c}(\alpha+\beta) \mathrm{s} \beta \mathrm{c} \kappa_{B i, 2}+\mathrm{s}(\alpha+\beta) \mathrm{c} \beta\right]
\end{array}\right)}
\end{aligned}
$$

对于球面机构 $C_{\mathrm{s} i}$

$$
\kappa_{C i, 1}=\eta_{i, 1}, \kappa_{C i, 2}=\kappa_{C i, 6}=\kappa_{B i, 2}, \kappa_{C i, 5}=\kappa_{C i, 3},
$$




$$
\begin{aligned}
& \tan \frac{\kappa_{C i, 3}}{2}=\frac{\left.\begin{array}{l}
\mathrm{s} \alpha \mathrm{c}(\alpha+\beta) \tan \frac{\kappa_{C i, 1}}{2} \mathrm{~s} \kappa_{C i, 2}+\mathrm{s} \alpha \mathrm{c} \kappa_{C i, 2}- \\
\left\{\left[\mathrm{s} \alpha \mathrm{c}(\alpha+\beta) \tan \frac{\kappa_{C i, 1}}{2} \mathrm{~s} \kappa_{C i, 2}+\mathrm{s} \alpha \mathrm{c} \kappa_{C i, 2}\right]^{2}-\right. \\
\mathrm{s}(\alpha+\beta)\left[\mathrm{c}(\alpha+\beta) \tan \frac{\kappa_{C i, 1}}{2}\left(\mathrm{c} \kappa_{C i, 2}+1\right)-\right. \\
\left.\mathrm{s} \kappa_{C i, 2}\right]\left[\mathrm{c}(\alpha+\beta) \mathrm{s}(\beta-\alpha) \tan \frac{\kappa_{C i, 1}}{2} \mathrm{c} \kappa_{C i, 2}+\right. \\
\mathrm{s}(\alpha+\beta) \mathrm{c}(\beta-\alpha) \tan \frac{\kappa_{C i, 1}}{2}- \\
\left.\mathrm{s}(\alpha+\beta)\left[\mathrm{s} \kappa_{C i, 2}\right]\right\}^{1 / 2}
\end{array}\right)}{\left.\mathrm{c}(\alpha+\beta) \tan \frac{\kappa_{C i, 1}}{2}\left(\mathrm{c} \kappa_{C i, 2}+1\right)-\mathrm{s} \kappa_{C i, 2}\right]} \\
& \tan \frac{\kappa_{C i, 4}}{2}=\frac{\left[\begin{array}{l}
\mathrm{s}(\alpha+\beta)\left(\mathrm{c} \beta \mathrm{c} \kappa_{C i, 2} \mathrm{~s} \kappa_{C i, 3}-\mathrm{s} \kappa_{C i, 2} \mathrm{c} \kappa_{C i, 3}\right)+ \\
\mathrm{c}(\alpha+\beta) \mathrm{s} \beta \mathrm{s} \kappa_{C i, 3}
\end{array}\right]}{\left(\begin{array}{l}
\mathrm{c} \alpha\left[\mathrm{s}(\alpha+\beta) \mathrm{c} \beta \mathrm{c} \kappa_{C i, 2} \mathrm{c} \kappa_{C i, 3}+\right. \\
\left.\mathrm{c}(\alpha+\beta) \mathrm{s} \beta \mathrm{c} \kappa_{C i, 3}+\mathrm{s}(\alpha+\beta) \mathrm{s} \kappa_{C i, 2} \mathrm{~s} \kappa_{C i, 3}\right]+ \\
\mathrm{s} \alpha\left[\mathrm{s}(\alpha+\beta) \mathrm{s} \beta \mathrm{c} \kappa_{C i, 2}-\mathrm{c}(\alpha+\beta) \mathrm{c} \beta\right]
\end{array}\right.}
\end{aligned}
$$

对于球面机构 $A_{\mathrm{s}(i+1)}$

$$
\begin{gathered}
\eta_{i+1,4}=\kappa_{B i, 1}, \eta_{i+1,3}=\eta_{i+1,5}=\eta_{C i, 3}, \eta_{i+1,2}=\eta_{i+1,6}, \\
\tan \frac{\eta_{i+1,6}}{2}=\frac{\left(\begin{array}{l}
\mathrm{c} \eta_{i+1,5}+\mathrm{c} \alpha \tan \frac{\eta_{i+1,4}}{2} \mathrm{~s} \eta_{i+1,5}-\left\{\left(\mathrm{c} \eta_{i+1,5}+\right.\right. \\
\left.\mathrm{c} \alpha \tan \frac{\eta_{i+1,4}}{2} \mathrm{~s} \eta_{i+1,5}\right)^{2}-2 \mathrm{c} \alpha \tan \frac{\eta_{i+1,4}}{2}\left[\mathrm{~s} \eta_{i+1,5}-\right. \\
\left.\mathrm{c} \alpha \tan \frac{\eta_{i+1,4}}{2}\left(\mathrm{c} \eta_{i+1,5}+1\right)\right]-\left(2 \mathrm{c}^{2} \alpha+\mathrm{c} 2 \alpha\right) . \\
\left.\left[\mathrm{s} \eta_{i+1,5}-\mathrm{c} \alpha \tan \frac{\eta_{i+1,4}}{2}\left(\mathrm{c} \eta_{i+1,5}+1\right)\right]^{2}\right\}^{1 / 2}
\end{array}\right)}{\tan \frac{\eta_{i+1,1}}{2}=\frac{\left[\begin{array}{l}
\mathrm{s} \alpha\left(\mathrm{s} \eta_{i+1,5} \mathrm{c} \eta_{i+1,6}-\mathrm{c} \alpha \tan \frac{\eta_{i+1,4}}{2}\left(\mathrm{c} \eta_{i+1,5}+1\right)\right. \\
\left.\mathrm{c} \alpha \mathrm{s} 2 \alpha \mathrm{s} \eta_{i+1,6} \mathrm{c} \eta_{i+1,5}\right)-
\end{array}\right]}{\left[\begin{array}{l}
\mathrm{s} \alpha\left(\mathrm{s} \alpha \mathrm{s} 2 \alpha \mathrm{c} \eta_{i+1,5}-\mathrm{c} \alpha \mathrm{c} 2 \alpha\right)- \\
\mathrm{c} \alpha\left(\mathrm{c} \alpha \mathrm{s} 2 \alpha \mathrm{c} \eta_{i+1,6}+\mathrm{s} \alpha \mathrm{s} \eta_{i+1,5} \mathrm{~s} \eta_{i+1,6}+\right. \\
\left.\mathrm{s} \alpha \mathrm{c} 2 \alpha \mathrm{c} \eta_{i+1,5} \mathrm{c} \eta_{i+1,6}\right)
\end{array}\right]}}
\end{gathered}
$$$$
i=1,2, \cdots, \frac{m-1}{2}
$$

从式(12)可以看出, 只需一个输入 $\eta_{0,1}$ 即可完全确 定整个 Waterbomb 折纸管的运动, 说明在给定的运动 对称条件下该折纸管的自由度为 1 。考虑到纵向的对 称性, 这里仅给出上半部分的运动方程, 下半部分的 运动方程同公式(12)，仅需改变对应层的编号。

为了找出 Waterbomb 折纸管刚性折叠时折叠角 度 $\eta_{0,1}$ 的范围, 我们需考虑以下三个方面的约束。
首先, Waterbomb 折纸管径向收缩运动的一个极限 为其中间层完全收缩构型(图 5a), 此时球面机构 $B_{\mathrm{s} 0}$ 处于完全折叠状态, 且 $\kappa_{B 0,4}=0$, 即公共折痕 $B_{0} C_{-1}$ 连接的相邻两个 Waterbomb 单元的三角形面板完全 重合。图中, $A_{i}^{\mathrm{p}}, B_{i}^{\mathrm{p}}, C_{i}^{\mathrm{p}}$ 分别为顶点 $A_{i}, B_{i}$ 和 $C_{i}$ 在赤道面上的投影。根据式(12b)可知, 此时

$$
\eta_{0,1}=\frac{2 \pi}{n}
$$

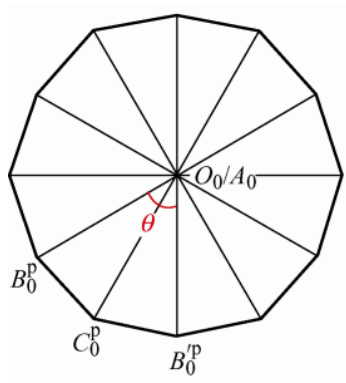

(a) 中间层完全收缩时, 0层在赤道面的投影

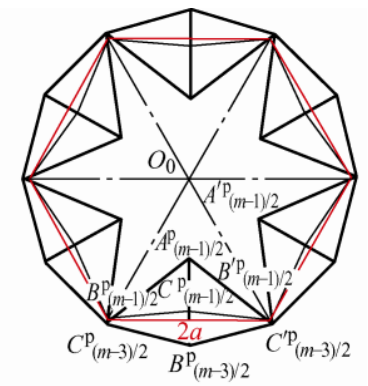

(b) 最顶层完全展开时, $(m-1) / 2$ 层在赤道面的投影
图 5 Waterbomb 折纸管径向收缩运动的极限位置

其次, 由于 Waterbomb 折纸管折叠时呈骨头状, 故径向收缩运动的另一极限为其最顶层完全展开构 型(图 5b), 此时第 $(m-1) / 2$ 层上所有 Waterbomb 单元 上方的对角顶点构成一个边长为 $2 a$ 的 $n$ 边形, 且 $\eta_{(m-1) / 2,1}=\pi$ 。令式(12g)中 $\tan \frac{\eta_{(m-1) / 2,6}}{2}$ 表达式里的平 方根为 0 , 有

$$
\begin{aligned}
& \frac{2 \pi}{n}\left(\mathrm{c} \eta_{(m-1) / 2,5}+\mathrm{c} \alpha \tan \frac{\eta_{(m-1) / 2,4}}{2} \mathrm{~s} \eta_{(m-1) / 2,5}\right)^{2}- \\
& 2 \mathrm{c} \alpha \tan \frac{\eta_{(m-1) / 2,4}}{2}\left[\mathrm{~s} \eta_{(m-1) / 2,5}-\mathrm{c} \alpha\left(\mathrm{c} \eta_{(m-1) / 2,5}+\right.\right. \\
& \left.1) \tan \frac{\eta_{(m-1) / 2,4}}{2}\right]-\left(2 \mathrm{c}^{2} \alpha+\mathrm{c} 2 \alpha\right)\left[\mathrm{s} \eta_{(m-1) / 2,5}-\right. \\
& \left.\operatorname{c} \alpha \tan \frac{\eta_{(m-1) / 2,4}}{2}\left(\mathrm{c} \eta_{(m-1) / 2,5}+1\right)\right]^{2}=0
\end{aligned}
$$

由此可求得 $\eta_{0,1}$ 的另一极限。然而, 由于式(14)的高 度非线性, $\eta_{0,1}$ 至多有三个解。因此, Waterbomb 折纸管刚性径向收缩运动的两个极限值中, $\eta_{0,1 \mathrm{~min}}$ 为 式(14)中 $\eta_{0,1}$ 解的最小值与式(13)中 $\eta_{0,1}$ 值相比的较 大值, 而 $\eta_{0,1 \max }$ 为式(14)中 $\eta_{0,1}$ 解的最大值。

最后, 为了避免 Waterbomb 折纸管在径向收缩过 程中面板间发生运动学干涉, 还需要满足以下条件

$$
\kappa_{B i, 4} \geqslant 0 \quad i=0,1, \cdots, \frac{m-1}{2}
$$

将式(12)代入式(15)中，可得到公式(15)的解。因此, 要使 Waterbomb 折纸管具有刚性径向收缩运动且不发 
生干涉, 其折叠角度 $\eta_{0,1}$ 的范围可由式(13) (15)确定。

以 $n=6, \alpha=40^{\circ}, \beta=35^{\circ}$ 的 Waterbomb 折纸 管为例, 当 $m=3$ 时, 由式(13) (15) 可求得其刚性 折叠范围为 $60^{\circ} \leqslant \eta_{0,1} \leqslant 120.6^{\circ}$, 此时 $\kappa_{B i, 4}$ 和 $\eta_{i, 1}(i=0,1)$ 与 $\eta_{0,1}$ 间的运动关系如图 6 所示, 其中 $\mathrm{i} \sim \mathrm{v}$ 为 $\eta_{0,1}$ 取不同角度时对应的管子构型, 构型 $\mathrm{i}$ 对应管子中间层完全折叠状态, 即 $\kappa_{B 0,4}=0$, 构型 $\mathrm{v}$ 对应管子最顶层完全展开状态, 即 $\eta_{1,1}=\pi$ 。当 $m=5$ 时, 管子的第 2 层不存在满足周向闭环条件的 解, 即此时该管子不存在刚性径向收缩运动。

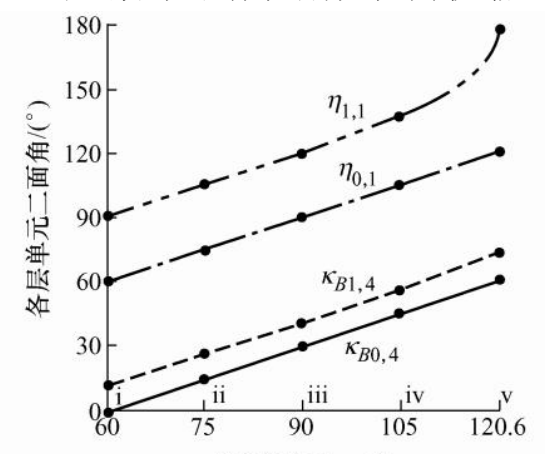

折叠角度 $\eta_{0.1} /\left(^{\circ}\right)$

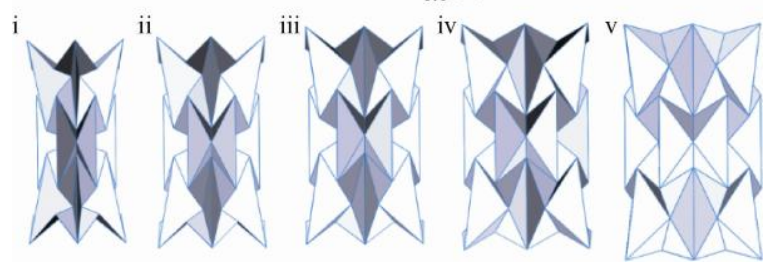

图 $6 m=3, n=6, \alpha=40^{\circ}, \beta=35^{\circ}$ 的 Waterbomb 折纸管的运动曲线及构型

若取 $n=6, \alpha=45^{\circ}, \beta=40^{\circ}$, 当 $m=3$ 时, 由式(13) (15)可求得 Waterbomb 折纸管的刚性折 叠范围为 $60^{\circ} \leqslant \eta_{0,1} \leqslant 139.8^{\circ}$ 。图 $7 \mathrm{a}$ 中的灰线给出 了此时 $\kappa_{B i, 4}$ 和 $\eta_{i, 1}(i=0,1)$ 与 $\eta_{0,1}$ 间的运动关系。此 时该管子的构型变化过程 $\mathrm{i} \sim \mathrm{v}$ 如图 $7 \mathrm{~b}$ 所示。构型 $\mathrm{i}$ 对应管子中间层完全折叠状态, 即 $\kappa_{B 0,4}=0$, 构型 $\mathrm{v}$ 对应管子最顶层完全展开状态, 即 $\eta_{1,1}=\pi$ 。若取 $m=5$, 由式(13) (15)可求得 Waterbomb 折纸管的 刚性折叠范围变成 $60^{\circ} \leqslant \eta_{0,1} \leqslant 131.1^{\circ}$, 此时 $\kappa_{B i, 4}$ 和 $\eta_{i, 1}(i=0,1,2)$ 与 $\eta_{0,1}$ 间的运动关系如图 $7 \mathrm{a}$ 中的黑线 所示。图 7c 给出了 $m=5$ 时的管子构型图 $\mathrm{I} \sim \mathrm{V}$ 。构 型 I 对应管子中间层完全折叠状态, 即 $\kappa_{B 0,4}=0$, 构型 $\mathrm{V}$ 对应管子最顶层完全展开状态，即 $\eta_{2,1}=\pi$ 。 由图 7a 可以看出, 随着管子层数的增加, 其运动曲 线的范围变小, 而角度关系保持不变, 即管子刚性 折叠运动范围变小, 而新增层 Waterbomb 单元的运 动不影响已有层单元的运动。保持 $n, \alpha$ 和 $\beta$ 不变, 该管子刚性运动范围与层数 $m$ 间的关系如图 $8 \mathrm{a}$ 所
示, 其中 $m$ 取从 3 到 15 的奇数。由图 $8 \mathrm{a}$ 可以看出, 当 $m$ 增大时, 该管子具有刚性运动的 $\theta$ (即 $\eta_{0,1}$ ) 的最 小值先不变后增大, 而最大值减小, 即其刚性运动 范围递减。

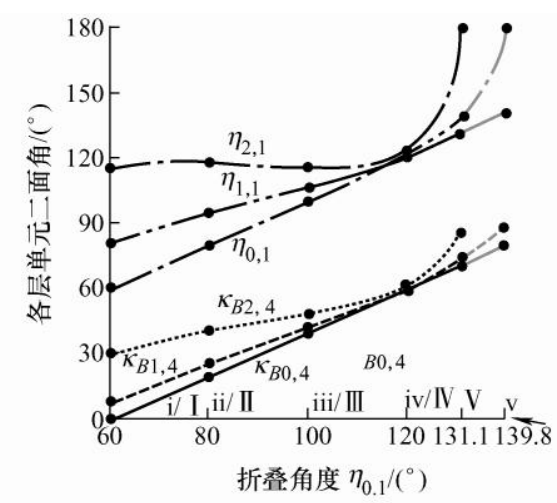

(a) 运动曲线图

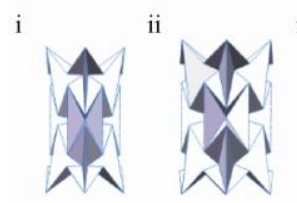

iil
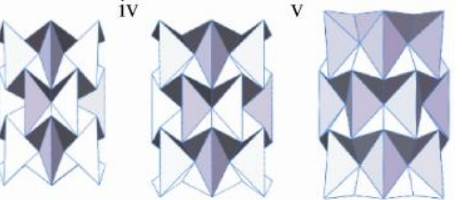

(b) $m=3$ 构型图
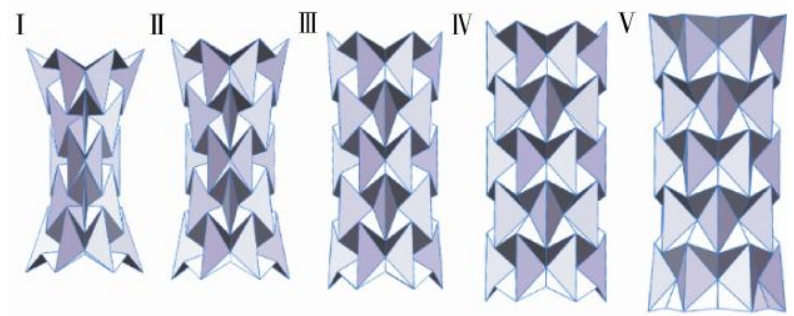

(c) $m=5$ 构型图

图 $7 n=6, \alpha=45^{\circ}, \beta=40^{\circ}$ 的 Waterbomb 折纸管的运动曲线及构型

当 $m=7, \alpha=45^{\circ}, \beta=40^{\circ}$ 时, 为使 Waterbomb 折纸管具有刚性径向收缩运动, $n \geqslant 6$ 。图 $8 \mathrm{~b}$ 给出 了此时该管子刚性运动范围与每层单元数 $n$ 间的关 系, 其中 $n$ 取从 6 到 20 的整数。由图 $8 b$ 可以看出, 当 $n$ 增大时, 该管子具有刚性运动的 $\theta_{\text {min }}$ 减小, 而 $\theta_{\text {max }}$ 增大, 其刚性运动范围递增。

若保持 $n=6, m=7, \quad \beta=40^{\circ}$ 不变, $\alpha$ 取 $20^{\circ} \leqslant \alpha \leqslant 70^{\circ}$, 该管子刚性运动范围与设计角度 $\alpha$ 间的关系如图 $8 \mathrm{c}$ 所示。由图 $8 \mathrm{c}$ 可以看出, 当 $\alpha \leqslant 31.3^{\circ}$ 时, 该管子的刚性运动范围递增。此时, 管子的最顶层发生物理干涉, 如图中红线所示, 发 生物理干涉的范围与 $\alpha$ 正相关。此时若 Waterbomb 管子要进行径向收缩运动, 必然存在面内变形, 该 管子存在结构变形-机构运动相互转换的混合模式。 当 $31.3^{\circ} \leqslant \alpha \leqslant 44.5^{\circ}$ 时, $\theta_{\text {min }}$ 不变, $\theta_{\text {max }}$ 先减小后增 大, 则该管子刚性运动范围也先减小后增大, 且其 发生物理干涉的范围逐渐减少, 直至 $\alpha=37.3^{\circ}$ 时不 
再存在物理干涉。进一步增大 $\alpha, \theta_{\text {min }}$ 先增大后减小 直至不变, $\theta_{\text {max }}$ 先增大后减小, 其刚性运动范围先减 小后增大再减小。当 $\alpha \geqslant 54.6^{\circ}$ 时, 该管子的中间层 将发生物理干涉, 且其干涉范围随着 $\alpha$ 增大而增大。

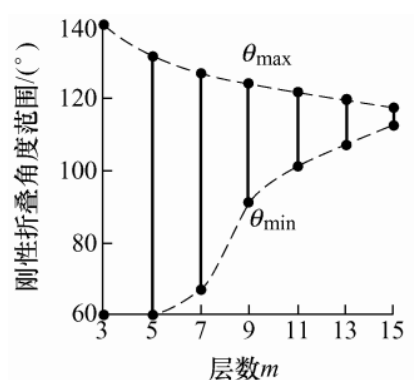

(a) 层数 $m$ 的影响

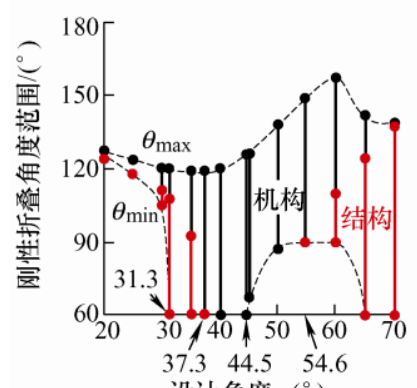

设计角度 $\left.\alpha{ }^{\circ}\right)$

(c) 设计角度 $\alpha$ 的影响

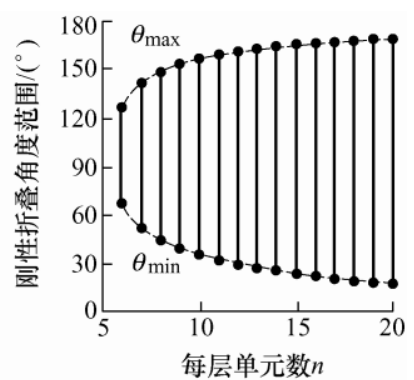

(b) 每层单元数 $n$ 的影响

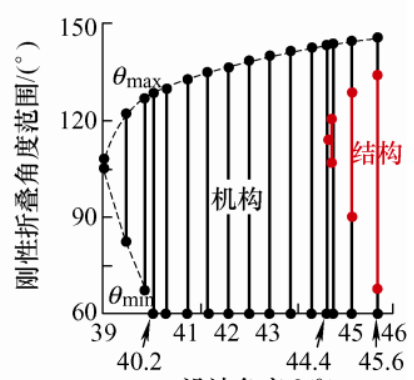

计角度 $\beta\left(^{\circ}\right)$
图 8 几何参数对 Waterbomb 折纸管径向收缩运动的影响

当 $n=6, m=7, \alpha=45^{\circ}$ 时, 为使 Waterbomb 折纸管具有刚性径向收缩运动, 设计角度 $\beta$ 的取值 范围为 $39^{\circ} \leqslant \beta \leqslant 45.6^{\circ}$, 且其与管子刚性运动范围 的关系如图 $8 \mathrm{~d}$ 所示。由图 $8 \mathrm{~d}$ 可以看出, 当 $39^{\circ} \leqslant \beta \leqslant 40.2^{\circ}$ 时, $\theta_{\text {min }}$ 减小, $\theta_{\text {max }}$ 增大, 该管子 刚性运动范围与 $\beta$ 正相关。当 $\beta \geqslant 40.2^{\circ}$ 时, $\theta_{\text {min }}$ 不 变, $\theta_{\text {max }}$ 增大, 该管子刚性运动范围递增。当 $\beta \geqslant 44.4^{\circ}$ 时, Waterbomb 管子最顶层发生物理干 涉, 且物理干涉的范围随着 $\beta$ 的增大而增大, 此时 管子存在机构运动-结构变形-机构运动相互转换的 混合模式。

\section{$2.2 \mathrm{~m}$ 为偶数}

此种情况下, Waterbomb 折纸管不存在线面对 称的中间层。如图 9a 所示, 赤道面穿过紧挨该面的 两层单元相连边的中点, 我们将这两层分别定义为 1 层和-1 层。所有单元仍具有面对称特性。因此, 式(12e) (12g)此时仍成立, 其中 $i=1,2, \cdots, \frac{m}{2}-1$ 。 由前述奇数层的分析可知, 一旦 -1 层和 1 层 Waterbomb 单元的运动确定, 其他层单元的运动即 可由公式(12e) $(12 \mathrm{~g})$ 得到。

与奇数层情况不同的是, 此时球面六杆机构 $A_{\mathrm{s} 0}$ 的运动不再满足线对称条件。考虑运动假设条件 3 ,
即该折纸管上下两部分的运动相同, 所以球面六杆 机构 $B_{\mathrm{s} 0}$ 和 $C_{\mathrm{s} 0}$ 的运动相同，可知

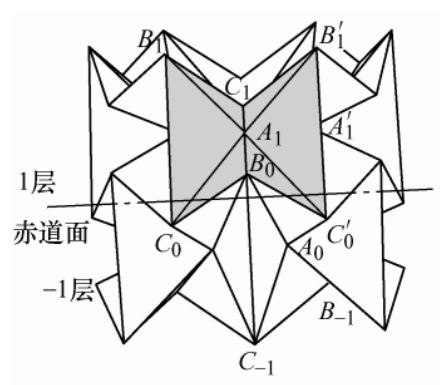

(a) 三维图

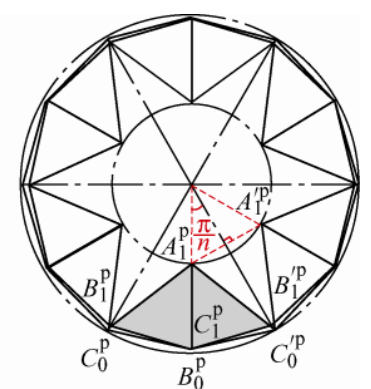

(b) 1 层在赤道面的投影
图 $9 m$ 为偶数的 Waterbomb 折纸管

$$
\begin{gathered}
\eta_{0,1}=\eta_{1,4} \\
\kappa_{B 0,1}=\kappa_{C 0,1}=\eta_{0,1}
\end{gathered}
$$

图 $9 \mathrm{~b}$ 给出了 1 层在赤道面的投影。为满足管状结构 的周向闭环条件, 以下方程需成立

$$
\mathrm{c} \kappa_{B 0,4}=\mathrm{c} \kappa_{C 0,4}=1-2\left(\frac{r_{A 1}}{a}\right)^{2} \mathrm{~s}^{2} \frac{\pi}{n}
$$

$$
\begin{aligned}
& r_{A 1}=r_{A 0}= \\
& r_{B 0}-\frac{[\cot \alpha-\cot (\alpha+\beta)] \mathrm{c} \frac{\eta_{0,1}}{2}}{\cot ^{2}(\alpha+\beta)+\mathrm{c}^{2} \frac{\eta_{0,1}}{2}} \cdot \\
& \sqrt{a^{2}\left[\cot ^{2}(\alpha+\beta)++\mathrm{c}^{2} \frac{\eta_{0,1}}{2}\right]-r_{B 0}^{2}\left(1-\mathrm{c} \frac{\pi}{n}\right)^{2}} \\
& r_{B 0}=a \mathrm{~s} \frac{\eta_{0,1}}{2} / \mathrm{s} \frac{\pi}{n}
\end{aligned}
$$

将式(18)代入式(17), 可求得 $\kappa_{B 0,4}$ 与 $\eta_{0,1}$ 间的关系为

$$
\begin{gathered}
c \kappa_{B 0,4}=\mathrm{c} \eta_{0,1}-\frac{\left(\begin{array}{l}
2 \mathrm{~s}^{2} \frac{\pi}{n}[\cot (\alpha+\beta)-\cot \alpha]^{2} \mathrm{c}^{2} \frac{\eta_{0,1}}{2}\left[\mathrm{c}^{2} \frac{\eta_{0,1}}{2}+\right. \\
\left.\cot ^{2}(\alpha+\beta)-\mathrm{s}^{2} \frac{\eta_{0,1}}{2} \tan \frac{2 \pi}{n}\right]
\end{array}\right)}{\left[\cot ^{2}(\alpha+\beta)+\mathrm{c}^{2} \frac{\eta_{0,1}}{2}\right]^{2}} \\
+\frac{2 \mathrm{~s} \frac{\pi}{n}[\cot \alpha-\cot (\alpha+\beta)] \mathrm{s} \eta_{0,1}}{\cot ^{2}(\alpha+\beta)+\mathrm{c}^{2} \frac{\eta_{0,1}}{2}} \cdot \\
\sqrt{\cot ^{2}(\alpha+\beta)+\mathrm{c}^{2} \frac{\eta_{0,1}}{2}-\mathrm{s}^{2} \frac{\eta_{0,1}}{2} \tan \frac{2 \pi}{n}}
\end{gathered}
$$

将式(16b)代入式(6), 可得 $\eta_{0,1}$ 及 $\kappa_{B 0,4}$ 与 $\kappa_{B 0,2}$ 及 $\kappa_{B 0,3}$ 间的函数关系为 


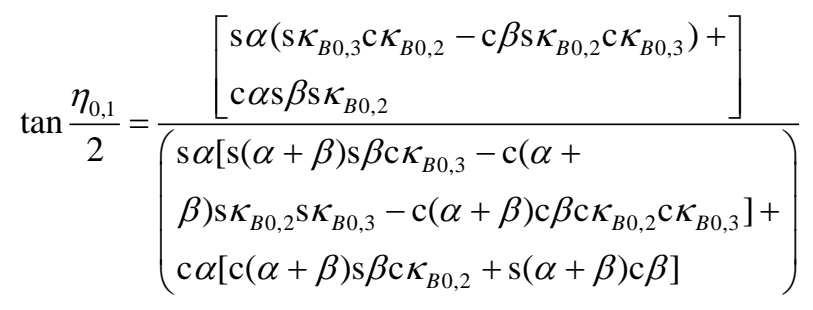

$$
\tan \frac{\kappa_{B 0,4}}{2}=\frac{\left[\begin{array}{l}
\mathrm{s}(\alpha+\beta)\left(\mathrm{c} \beta \mathrm{s} \kappa_{B 0,3} \mathrm{c} \kappa_{B 0,2}-\mathrm{s} \kappa_{B 0,2} \mathrm{c} \kappa_{B 0,3}\right)+ \\
\mathrm{c}(\alpha+\beta) \mathrm{s} \beta \mathrm{s} \kappa_{B 0,3}
\end{array}\right]}{\left(\begin{array}{l}
\mathrm{c} \alpha\left[\mathrm{s}(\alpha+\beta) \mathrm{c} \beta \mathrm{c} \kappa_{B 0,2} \mathrm{c} \kappa_{B 0,3}+\mathrm{c}(\alpha+\right. \\
\left.\beta) \mathrm{s} \beta \mathrm{c} \kappa_{B 0,3}+\mathrm{s}(\alpha+\beta) \mathrm{s} \kappa_{B 0,2} \mathrm{~s} \kappa_{B 0,3}\right]+ \\
\mathrm{s} \alpha\left[\mathrm{s}(\alpha+\beta) \mathrm{s} \beta \mathrm{c} \kappa_{B 0,2}-\mathrm{c}(\alpha+\beta) \mathrm{c} \beta\right]
\end{array}\right)}
$$

消去式(20a)、(20b)中的 $\kappa_{B 0,3}$ 项, 可得

$$
\begin{aligned}
& U_{1} \tan ^{6} \frac{\kappa_{B 0,2}}{2}+U_{2} \tan ^{5} \frac{\kappa_{B 0,2}}{2}+U_{3} \tan ^{4} \frac{\kappa_{B 0,2}}{2}+U_{7}+ \\
& U_{4} \tan ^{3} \frac{\kappa_{B 0,2}}{2}+U_{5} \tan ^{2} \frac{\kappa_{B 0,2}}{2}+U_{6} \tan \frac{\kappa_{B 0,2}}{2}=0
\end{aligned}
$$

式中, $U_{i}(i=1,2, \cdots, 7)$ 为关于 $\eta_{0,1}$ 与 $\kappa_{B 0,4}$ 的函数, 具体有

$$
\begin{aligned}
& U_{1}=4 \mathrm{~s} \alpha \mathrm{s} 2 \alpha\left(\tan ^{2} \frac{\kappa_{B 0,4}}{2}-\tan ^{2} \frac{\eta_{0,1}}{2}\right) \\
& U_{2}=16 \mathrm{~s} 2 \alpha \mathrm{s} \beta \tan \frac{\eta_{0,1}}{2}\left(\tan ^{2} \frac{\kappa_{B 0,4}}{2}+1\right)
\end{aligned}
$$$$
U_{3}=-\left\{\left[16 \mathrm{~s} 2 \alpha \mathrm{s} \beta \mathrm{c}(\alpha+\beta) \tan ^{2} \frac{\eta_{0,1}}{2}+11 \mathrm{c} \alpha+\right.\right.
$$$$
5 \mathrm{c} 3 \alpha+\mathrm{c}(3 \alpha+2 \beta)-7 \mathrm{c}(\alpha-2 \beta)-
$$$$
10 \mathrm{c}(\alpha+2 \beta)] \tan ^{2} \frac{\kappa_{B 0,4}}{2}+2 \mathrm{~s} \alpha[5 \mathrm{~s} 2(\alpha+\beta)+
$$$$
\left.\mathrm{s} 2 \alpha+3 \mathrm{~s} 2 \beta] \tan ^{2} \frac{\eta_{0,1}}{2}+32 \mathrm{c} \alpha \mathrm{s}^{2} \beta\right\}
$$$$
U_{4}=16 \mathrm{~s} \beta \mathrm{s}(\alpha+\beta)[3 \mathrm{c}(\alpha+\beta)+
$$$$
\mathrm{c}(\alpha-\beta)] \tan \frac{\eta_{0,1}}{2}\left(\tan ^{2} \frac{\kappa_{B 0,4}}{2}+1\right)
$$

$U_{5}=-4\left\{\left[4 \mathrm{~s} 2 \beta \mathrm{c}(\alpha+\beta) \mathrm{s}(2 \alpha+\beta) \tan ^{2} \frac{\eta_{0,1}}{2}-\right.\right.$

$\mathrm{s} 2 \alpha \mathrm{s}(\alpha+2 \beta)-2 \mathrm{~s} 2(\alpha+\beta) \mathrm{s}(\alpha-2 \beta)] \tan ^{2} \frac{\kappa_{B 0,4}}{2}+$

$2 \mathrm{~s}(\alpha+2 \beta)[\mathrm{s} 2(\alpha+\beta)+\mathrm{s} \alpha \mathrm{c} \alpha] \tan ^{2} \frac{\eta_{0,1}}{2}+$

$4 \mathrm{~s} \beta \mathrm{s} 2 \beta \mathrm{c}(\alpha+\beta)\}$

$U_{6}=16 \mathrm{~s} 2 \beta \mathrm{s}(\alpha+2 \beta) \mathrm{c}(\alpha+\beta) \tan \frac{\eta_{0,1}}{2}\left(\tan ^{2} \frac{\kappa_{B 0,4}}{2}+1\right)$

$U_{7}=-8 \mathrm{c} \beta \mathrm{c}(\alpha+\beta)\left\{\left[\mathrm{s} 2(\alpha+\beta) \mathrm{s} 2 \beta \tan ^{2} \frac{\eta_{0,1}}{2}-\right.\right.$

$$
\left.\left.\mathrm{s}^{2} \alpha\right] \tan ^{2} \frac{\kappa_{B 0,4}}{2}+\mathrm{s}^{2}(\alpha+2 \beta) \tan ^{2} \frac{\eta_{0,1}}{2}\right\}
$$

由式(21)可求得 $\kappa_{B 0,2}$, 且

$$
\tan \frac{\kappa_{B 0,3}}{2}=\frac{\mathrm{s} \alpha\left(\tan \frac{\kappa_{B 0,1}}{2}-\mathrm{c} \kappa_{B 0,2} \tan \frac{\kappa_{B 0,4}}{2}\right)-\mathrm{c} \alpha \mathrm{s} \kappa_{B 0,2}}{\mathrm{~s} \kappa_{B 0,2} \tan \frac{\kappa_{B 0,4}}{2}},
$$$$
\kappa_{B 0,5}=\kappa_{B 0,3} \quad \kappa_{B 0,6}=\kappa_{B 0,2} ;
$$

$$
\eta_{0,2}=\eta_{0,6}=\kappa_{B 0,3} \quad \eta_{0,3}=\eta_{0,5},
$$

$$
\tan \frac{\eta_{0,5}}{2}=\frac{\left(\begin{array}{l}
\left\{( 2 \mathrm { c } ^ { 2 } \alpha + \mathrm { c } 2 \alpha ) \left[\mathrm{s} \eta_{0,6}-\tan \frac{\eta_{0,1}}{2} \mathrm{c} \alpha\left(\mathrm{c} \eta_{0,6}+\right.\right.\right. \\
1)]^{2}+2 \mathrm{c} \alpha \tan \frac{\eta_{0,1}}{2}\left[\mathrm{~s} \eta_{0,6}-\tan \frac{\eta_{0,1}}{2} \mathrm{c} \alpha\left(\mathrm{c} \eta_{0,6}+\right.\right. \\
\left.1)]-\left(\tan \frac{\eta_{0,1}}{2} \mathrm{c} \alpha \mathrm{s} \eta_{0,6}+\mathrm{c} \eta_{0,6}\right)^{2}\right\}^{1 / 2}+\mathrm{c} \eta_{0,6}+ \\
\tan \frac{\eta_{0,1}}{2} \mathrm{c} \alpha \mathrm{s} \eta_{0,6}
\end{array}\right.}{\mathrm{s} \eta_{0,6}-\tan \frac{\eta_{0,1}}{2} \mathrm{c} \alpha\left(\mathrm{c} \eta_{0,6}+1\right)}
$$

$$
\tan \frac{\eta_{0,4}}{2}=\frac{\left[\begin{array}{l}
\mathrm{s} \alpha\left(\mathrm{s} \eta_{0,6} \mathrm{c} \eta_{0,5}-\mathrm{c} 2 \alpha \mathrm{s} \eta_{0,5} \mathrm{c} \eta_{0,6}\right)- \\
\mathrm{c} \alpha \mathrm{s} 2 \alpha \mathrm{s} \eta_{0,5}
\end{array}\right]}{\left[\begin{array}{l}
\mathrm{s} \alpha\left(\mathrm{s} \alpha \mathrm{s} 2 \alpha \mathrm{c} \eta_{0,6}-\mathrm{c} \alpha \mathrm{c} 2 \alpha\right)- \\
\mathrm{c} \alpha\left(\mathrm{c} \alpha \mathrm{s} 2 \alpha \mathrm{c} \eta_{0,5}+\mathrm{s} \alpha \mathrm{s} \eta_{0,6} \mathrm{~s} \eta_{0,5}\right. \\
\left.\mathrm{s} \alpha \mathrm{c} 2 \alpha \mathrm{c} \eta_{0,6} \mathrm{c} \eta_{0,5}\right)
\end{array}\right]}
$$

$\kappa_{C 0,1}=\kappa_{B 0,1} \quad \kappa_{C 0,2}=\kappa_{C 0,6}=\kappa_{B 0,2} \quad \kappa_{C 0,3}=\kappa_{C 0,5}=\kappa_{B 0,3}$

$$
\kappa_{C 0,4}=\kappa_{B 0,4}
$$

$$
\eta_{1,1}=\eta_{0,4} \quad \eta_{1,2}=\eta_{1,6}=\eta_{0,5} \quad \eta_{1,3}=\eta_{1,5}=\eta_{0,6}
$$

$$
\eta_{1,4}=\eta_{0,1}
$$

因此, 式 $(12 \mathrm{e}) \sim(12 \mathrm{~g}),(19) \sim(22)$ 构成了 $m$ 为 偶数条件下 Waterbomb 折纸管的运动学方程组。从 中可以看出, 只要知道变量 $\eta_{0,1}$ 即可完全确定该结 构的运动, 即此时 Waterbomb 折纸管的自由度也 为 1 。

同理, 我们需确定刚性运动的范围。与奇数层 情况不同的是, 这里我们仅需考虑两方面的约束,

即 $\eta_{m / 2,1}=\pi$ 和 $\kappa_{B i, 4} \geqslant 0\left(i=0,1, \cdots, \frac{m}{2}\right)$ 。这主要是因 为在偶数层条件下, -1 层和 1 层 Waterbomb 单元一 般不会同时达到完全折叠的状态。只有一种情况例 外, 即在特定几何条件下, 所有层单元同时到达完 全折叠状态。此时, 奇数层和偶数层的完全折叠构 型相同, 此处我们暂不考虑这种情形。 $\eta_{0,1 \mathrm{~min}}$ 可以通 过令式(19) 中的 $\kappa_{B 0,4}>0$ 得到。另一方面, 当 $\eta_{m / 2,1}=\pi$ 时, 令式(12g)中 $\tan \frac{\eta_{m / 2,6}}{2}$ 表达式里的平 方根为 0 , 有 
$\left(\mathrm{c} \eta_{m / 2,5}+\mathrm{c} \alpha \tan \frac{\eta_{m / 2,4}}{2} \mathrm{~s} \eta_{m / 2,5}\right)^{2}-2 \mathrm{c} \alpha \tan \frac{\eta_{m / 2,4}}{2}\left[\mathrm{~s} \eta_{m / 2,5}-\right.$ $\left.\mathrm{c} \alpha \tan \frac{\eta_{m / 2,4}}{2}\left(\mathrm{c} \eta_{m / 2,5}+1\right)\right]-\left(2 \mathrm{c}^{2} \alpha+\mathrm{c} 2 \alpha\right)\left[\mathrm{s} \eta_{m / 2,5}-\right.$ $\left.\operatorname{c} \alpha \tan \frac{\eta_{m / 2,4}}{2}\left(\mathrm{c} \eta_{m / 2,5}+1\right)\right]^{2}=0$

由式(12)可知, $\eta_{m / 2,5}$ 和 $\eta_{m / 2,4}$ 均是 $\eta_{0,1}$ 的函数, 故可 由式(23)求得 $\eta_{0,1 \max }$ 。因此, Waterbomb 折纸管没有 干涉的刚性收缩运动范围为 $\eta_{0,1 \text { min }}$ 与 $\eta_{0,1 \text { max }}$ 间满足 $\kappa_{B i, 4} \geqslant 0\left(i=0,1, \cdots, \frac{m}{2}\right)$ 的区间。

因此,一旦 Waterbomb 折纸管的几何参数给定, 即可确定其发生刚性收缩运动时 $\eta_{0,1}$ 的取值范围。 这一范围不仅与设计角度 $\alpha$ 和 $\beta$ 有关, 还与单元层 数 $m$ 和每层单元数 $n$ 相关。另一方面, 我们也可以 通过令 Waterbomb 折纸管的运动学方程有解来获得 存在刚性收缩运动情况下几何参数 $\alpha$ 和 $\beta$ 的范围。

\section{3 扭转运动}

在对 $\alpha=\beta=45^{\circ}$ 的 Waterbomb 折纸管的分析 中, 我们发现当奇数层的折纸管中间层完全折叠时, 该管子存在着刚性扭转运动 ${ }^{[27]}$ 。我们推断此运动对 于广义 Waterbomb 折纸管同样成立, 且扭转运动的 触发条件为该管子中间层单元到达完全折叠构型。 如图 10a 所示, 此时 $\kappa_{B 0,4}=0, r_{A 0}=0$, Waterbomb 管子处于刚性收缩运动的一个极限状态, 中间层单 元做线面对称运动。当扭转运动开始时, 球面六杆 机构 $A_{\mathrm{s} 0}$ 做线对称运动, 且

$$
\delta_{0,1}=\delta_{0,4}, \quad \delta_{0,2}=\delta_{0,5}, \quad \delta_{0,3}=\delta_{0,6}
$$

将式(24)代入闭环方程式(1)中, 可得

$$
\tan \delta_{0,1}=\frac{2 \mathrm{c} \alpha\left[\mathrm{s} \delta_{0,2}+\mathrm{s} \delta_{0,3}-\mathrm{s}\left(\delta_{0,2}+\delta_{0,3}\right)\right]}{\left[\begin{array}{l}
\left(1+2 \mathrm{c}^{2} \alpha\right) \mathrm{c} \delta_{0,2} \mathrm{c} \delta_{0,3}-\mathrm{s} \delta_{0,2} \mathrm{~s} \delta_{0,3}- \\
2 \mathrm{c}^{2} \alpha\left(\mathrm{c} \delta_{0,2}+\mathrm{c} \delta_{0,3}\right)+\mathrm{c} 2 \alpha
\end{array}\right]}
$$

将运动变量 $\delta_{0, i}$ 与二面角 $\eta_{0, i}$ 间的关系代入式(24)、 (25)中, 可得中间层 Waterbomb 单元二面角间的关 系为

$$
\begin{array}{r}
\tan \eta_{0,1}=\frac{2 \mathrm{c} \alpha\left[\mathrm{s} \eta_{0,2}+\mathrm{s} \eta_{0,3}+\mathrm{s}\left(\eta_{0,2}+\eta_{0,3}\right)\right]}{\left[\begin{array}{l}
\left(1+2 \mathrm{c}^{2} \alpha\right) \mathrm{c} \eta_{0,2} \mathrm{c} \eta_{0,3}-\mathrm{s} \eta_{0,2} \mathrm{~s} \eta_{0,3}+ \\
2 \mathrm{c}^{2} \alpha\left(\mathrm{c} \eta_{0,2}+\mathrm{c} \eta_{0,3}\right)+\mathrm{c} 2 \alpha
\end{array}\right]} \\
\eta_{0,4}=\eta_{0,1}, \quad \eta_{0,5}=\eta_{0,2}, \quad \eta_{0,6}=\eta_{0,3}
\end{array}
$$

为了使球面六杆机构 $A_{\mathrm{s} 0}$ 构成周向闭环, 需满 足 $\angle E A_{0} E^{\prime}=\frac{2 \pi}{n}$, 如图 $10 \mathrm{~b}$ 所示, 其中 $E$ 和 $E^{\prime}$ 分别 是折痕 $B_{0} C_{-1}$ 和 $B_{0}^{\prime} C_{-1}^{\prime}$ 的中点。以 $A_{0}$ 为原点建立坐标 系, $x$ 轴沿 $\boldsymbol{A}_{0} \boldsymbol{E}$ 方向, $z$ 轴垂直于面 $E A_{0} E^{\prime}, y$ 轴由 右手定则确定。过 $E$ 和 $E^{\prime}$ 点分别作 $A_{0} B_{0}$ 和 $A_{0} B_{0}^{\prime}$ 的 垂线, 垂足分别为 $D$ 和 $D^{\prime}$ 点。再分别过 $D$ 和 $D^{\prime}$ 点 在面 $A_{0} B_{0} C_{0}$ 和 $A_{0} B_{0}^{\prime} C_{0}$ 内作 $A_{0} B_{0}$ 和 $A_{0} B_{0}^{\prime}$ 的垂线。由 于 Waterbomb 单元几何的对称性, 这两垂线交于 $A_{0} C_{0}$ 折痕上的 $F$ 点, 即 $D F \perp A_{0} B_{0}, D^{\prime} F \perp A_{0} B_{0}^{\prime}$ 。 由此可知

$$
\eta_{0,2}=\angle F D^{\prime} E^{\prime} \quad \eta_{0, \sigma}=\angle F D L
$$

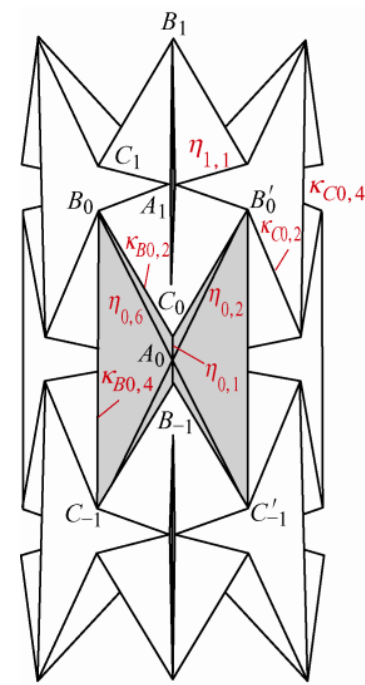

(a) 扭转运动的触发状态

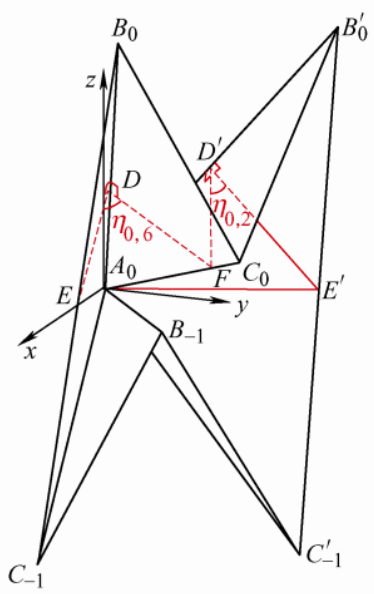

（b）中间层线对称球面机构 $A_{\mathrm{S} 0}$ 的几何
图 10 Waterbomb 折纸管的扭转运动

因为 $\angle E A_{0} E=\frac{2 \pi}{n}$, 点 $E$ 和 $E^{\prime}$ 的坐标分别为 $(a, 0,0)$ 和 $\left(a \mathrm{c} \frac{2 \pi}{n}, a \mathrm{~s} \frac{2 \pi}{n}, 0\right)$ 。假设点 $B_{0}, D, F$, $B_{0}^{\prime}$ 和 $D^{\prime}$ 的坐标分别为 $\left(x_{B 0}, y_{B 0}, z_{B 0}\right)$, $\left(x_{D}, y_{D}, z_{D}\right),\left(x_{F}, y_{F}, z_{F}\right),\left(x_{B^{\prime} 0}, y_{B^{\prime} 0}, z_{B^{\prime} 0}\right)$ 和 $\left(x_{D^{\prime}}, y_{D^{\prime}}, z_{D^{\prime}}\right)$, 可求得以下矢量为

$$
\begin{gathered}
\boldsymbol{E D}=\left(x_{D}-a, y_{D}, z_{D}\right), \\
\boldsymbol{F} \boldsymbol{D}=\left(x_{D}-x_{F}, y_{D}-y_{F}, z_{D}-z_{F}\right), \\
\boldsymbol{E}^{\prime} \boldsymbol{D}^{\prime}=\left(x_{D^{\prime}}-a \mathrm{c} \frac{2 \pi}{n}, y_{D^{\prime}}-a \mathrm{~s} \frac{2 \pi}{n}, z_{D^{\prime}}\right), \\
\boldsymbol{F} \boldsymbol{D}^{\prime}=\left(x_{D^{\prime}}-x_{F}, y_{D^{\prime}}-y_{F}, y_{D^{\prime}}-z_{F}\right)
\end{gathered}
$$

因为 $\overline{A_{0} D}=\overline{A_{0} D^{\prime}}=a \mathrm{~s} \alpha, \overline{D E}=\overline{D^{\prime} E^{\prime}}=a \mathrm{c} \alpha$, $\overline{D F}=\overline{D^{\prime} F}=a \mathrm{~s}^{2} \alpha / \mathrm{c} \alpha, \quad \overline{A_{0} B_{0}}=\overline{A_{0} B_{0}^{\prime}}=a / \mathrm{s} \alpha$, $\overline{B_{0} F}=\overline{B_{0}^{\prime} F}=a \sqrt{1-3 \mathrm{~s}^{2} \alpha+3 \mathrm{~s}^{4} \alpha} /(\mathrm{s} \alpha \mathrm{c} \alpha)$, 可知

$$
x_{D}^{2}+y_{D}^{2}+z_{D}^{2}=a^{2} \mathrm{~s}^{2} \alpha \quad x_{D^{\prime}}^{2}+y_{D^{\prime}}^{2}+z_{D^{\prime}}^{2}=a^{2} \mathrm{~s}^{2} \alpha
$$




$$
\begin{gathered}
\left(x_{D}-a\right)^{2}+y_{D}^{2}+z_{D}^{2}=a^{2} \mathrm{c}^{2} \alpha \\
\left(x_{D^{\prime}}-a \mathrm{c} \frac{2 \pi}{n}\right)^{2}+\left(y_{D^{\prime}}-a \mathrm{~s} \frac{2 \pi}{n}\right)^{2}+z_{D^{\prime}}^{2}=a^{2} \mathrm{c}^{2} \alpha \\
x_{B 0}^{2}+y_{B 0}^{2}+z_{B 0}^{2}=\frac{a^{2}}{\mathrm{~s}^{2} \alpha} \quad x_{B^{\prime} 0}^{2}+y_{B^{\prime} 0}^{2}+z_{B^{\prime} 0}^{2}=\frac{a^{2}}{\mathrm{~s}^{2} \alpha} \\
x_{D^{2}} x_{F}+y_{D} y_{F}+z_{D} z_{F}=a^{2} \mathrm{~s}^{2} \alpha \\
x_{D^{\prime}} x_{F}+y_{D^{\prime}} y_{F}+z_{D^{\prime}} z_{F}=a^{2} \mathrm{~s}^{2} \alpha \\
x_{B 0} x_{F}+y_{B 0} y_{F}+z_{B 0} z_{F}=a^{2} \\
x_{B^{\prime} 0} x_{F}+y_{B^{\prime} 0} y_{F}+z_{B^{\prime} 0} z_{F}=a^{2}
\end{gathered}
$$

此外, 由于 $A_{0} E \perp B_{0} E, \overline{B_{0} E}=\overline{B_{0}^{\prime} E^{\prime}}=a \mathrm{c} \alpha / \mathrm{s} \alpha$, 可得

$$
x_{B 0}=a \quad \mathrm{c} \frac{2 \pi}{n} x_{B^{\prime} 0}+\mathrm{s} \frac{2 \pi}{n} y_{B^{\prime} 0}=a
$$

根据 Waterbomb 单元的线对称性, $B_{0}$ 和 $B_{0}^{\prime}$ 点 $y$ 坐 标间的关系为

$$
y_{B 0}=\left(y_{B^{\prime} 0}-a \mathrm{~s} \frac{2 \pi}{n}\right) / \mathrm{c} \frac{2 \pi}{n}
$$

将式(30)、(31)代入式(29)中，可得

$$
z_{B 0}=z_{B^{\prime} 0}=\frac{\sqrt{-y_{B^{\prime} 0}^{2}+2 a \mathrm{~s} \frac{2 \pi}{n} y_{B^{\prime} 0}+a^{2}\left(\mathrm{c}^{2} \frac{2 \pi}{n} / \mathrm{s}^{2} \alpha-1\right)}}{\mathrm{c} \frac{2 \pi}{n}}
$$

$\left[\left(1-\mathrm{c} \frac{2 \pi}{n}\right) y_{B^{\prime} 0}-a \mathrm{~s} \frac{2 \pi}{n}\right] y_{F}=\left[\left(1-\mathrm{c} \frac{2 \pi}{n}\right) a-\mathrm{s} \frac{2 \pi}{n} y_{B^{\prime} 0}\right] x_{F}$

联立式(29) (32)并采用余弦定理，可知

$$
\begin{gathered}
x_{F}=a \mathrm{~s}^{2} \alpha\left(\mathrm{c} \eta_{0,6}+1\right) \\
y_{F}=a \mathrm{~s}^{2} \alpha\left(\mathrm{c} \eta_{0,2}-\mathrm{c} \frac{2 \pi}{n} \mathrm{c} \eta_{0,6}+1-\mathrm{c} \frac{2 \pi}{n}\right) / \mathrm{s} \frac{2 \pi}{n} \\
z_{F}=\frac{\left(\begin{array}{l}
a \mathrm{~s} \alpha\left[-\mathrm{s}^{2} \alpha\left(\mathrm{c}^{2} \eta_{0,2}+\mathrm{c}^{2} \eta_{0,6}-2 \mathrm{c} \frac{2 \pi}{n} \mathrm{c} \eta_{0,2} \mathrm{c} \eta_{0,6}\right)+\right. \\
\left.\left(1-\mathrm{c} \frac{2 \pi}{n}\right)\left(\mathrm{c} 2 \alpha \mathrm{c} \eta_{0,2}+\mathrm{c} 2 \alpha \mathrm{c} \eta_{0,6}+2 \mathrm{c}^{2} \alpha\right)\right]
\end{array}\right)}{\left(\begin{array}{l}
\left(1-\mathrm{c} \frac{2 \pi}{n}\right)\left[\left(\mathrm{c} 2 \alpha-\mathrm{c} \frac{2 \pi}{n}\right)\left(\mathrm{c}^{2} \eta_{0,2}+\mathrm{c}^{2} \eta_{0,6}\right)+\right)^{1 / 2} \\
2\left(1-\mathrm{c} 2 \alpha \mathrm{c} \frac{2 \pi}{n}\right) \mathrm{c} \eta_{0,2} \mathrm{c} \eta_{0,6}+ \\
\left.4 \mathrm{c}^{2} \alpha\left(1-\mathrm{c} \frac{2 \pi}{n}\right)\left(\mathrm{c} \eta_{0,2}+\mathrm{c} \eta_{0,6}+1\right)\right]
\end{array}\right)^{1}}
\end{gathered}
$$

由于 $\overline{A_{0} F}=a \tan \alpha$, 我们可求得 $\eta_{0,2}$ 和 $\eta_{0,6}$ 间的关系为

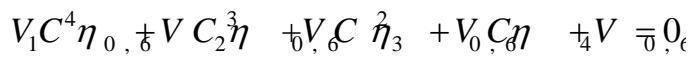

其中, $V_{i}(i=1,2, \cdots, 5)$ 为 $\eta_{0,2}$ 的函数, 具体有
$V_{1}=\mathrm{s}^{2} \alpha \mathrm{c}^{4} \alpha$

$V_{2}=2 \mathrm{~s}^{2} \alpha \mathrm{c}^{2} \alpha\left[\left(1-\mathrm{c} 2 \alpha \mathrm{c} \frac{2 \pi}{n}\right) \mathrm{c} \eta_{0,2}+2 \mathrm{c}^{2} \alpha\left(1-\mathrm{c} \frac{2 \pi}{n}\right)\right]$

$V_{3}=2 \mathrm{~s}^{2} \alpha \mathrm{c}^{2} \alpha\left(\mathrm{c}^{2} \alpha-2 \mathrm{~s}^{2} \alpha \mathrm{c}^{2} \frac{2 \pi}{n}-2 \mathrm{c} \frac{2 \pi}{n}\right) \mathrm{c}^{2} \eta_{0,2}+$ $4 \mathrm{~s}^{2} \alpha \mathrm{c}^{2} \alpha\left(1-\mathrm{c} \frac{2 \pi}{n}\right)\left(\mathrm{c}^{2} \alpha+1-\mathrm{c} 2 \alpha \mathrm{c} \frac{2 \pi}{n}\right) \mathrm{c} \eta_{0,2}+$ $\mathrm{s}^{2} \alpha\left(4 \mathrm{c}^{4} \alpha+1\right) \mathrm{c}^{2} \frac{2 \pi}{n}+2\left(6 \mathrm{c}^{6} \alpha-5 \mathrm{c}^{4} \alpha-\mathrm{c} 2 \alpha\right) \mathrm{c} \frac{2 \pi}{n}-$ $8 \mathrm{c}^{6} \alpha+10 \mathrm{c}^{4} \alpha-3 \mathrm{c}^{2} \alpha+1$ $V_{4}=2 \mathrm{~s}^{2} \alpha \mathrm{c}^{2} \alpha\left(1-\mathrm{c} 2 \alpha \mathrm{c} \frac{2 \pi}{n}\right) \mathrm{c}^{3} \eta_{0,2}+4 \mathrm{~s}^{2} \alpha \mathrm{c}^{2} \alpha\left(\mathrm{c}^{2} \alpha+1-\right.$ $\left.\mathrm{c} 2 \alpha \mathrm{c} \frac{2 \pi}{n}\right)\left(1-\mathrm{c} \frac{2 \pi}{n}\right) \mathrm{c}^{2} \eta_{0,2}+2\left[\mathrm{~s}^{2} \alpha\left(8 \mathrm{c}^{4} \alpha-1\right) \mathrm{c}^{2} \frac{2 \pi}{n}+\right.$ $2\left(6 c^{6} \alpha-7 c^{4} \alpha+2 c^{2} \alpha-1\right) c \frac{2 \pi}{n}-4 c^{6} \alpha+2 c^{4} \alpha+3 c^{2} \alpha-$ 1]c $\eta_{0,2}+4 \mathrm{~s}^{2} \alpha \mathrm{c}^{2} \alpha\left(1-\mathrm{c} \frac{2 \pi}{n}\right)\left[\mathrm{c} 2 \alpha-\left(1+2 \mathrm{c}^{2} \alpha\right) \mathrm{c} \frac{2 \pi}{n}\right]$ $V_{5}=\mathrm{s}^{2} \alpha \mathrm{c}^{4} \alpha \mathrm{c}^{4} \eta_{0,2}+4 \mathrm{~s}^{2} \alpha \mathrm{c}^{4} \alpha\left(1-\mathrm{c} \frac{2 \pi}{n}\right) \mathrm{c}^{3} \eta_{0,2}+$ $\left[\mathrm{s}^{2} \alpha\left(4 \mathrm{c}^{4} \alpha+1\right) \mathrm{c}^{2} \frac{2 \pi}{n}+2\left(6 \mathrm{c}^{6} \alpha-\mathrm{c} 2 \alpha-5 \mathrm{c}^{4} \alpha\right) \mathrm{c} \frac{2 \pi}{n}-\right.$ $\left.8 \mathrm{c}^{6} \alpha+10 \mathrm{c}^{4} \alpha-3 \mathrm{c}^{2} \alpha+1\right] \mathrm{c}^{2} \eta_{0,2}+4 \mathrm{~s}^{2} \alpha \mathrm{c}^{2} \alpha[\mathrm{c} 2 \alpha-$ $\left.\left(1+2 \mathrm{c}^{2} \alpha\right) \mathrm{c} \frac{2 \pi}{n}\right]\left(1-\mathrm{c} \frac{2 \pi}{n}\right) \mathrm{c} \eta_{0,2}-4 \mathrm{~s}^{2} \alpha \mathrm{c}^{2} \alpha(1-$ $\left.\mathrm{c} \frac{2 \pi}{n}\right)\left[\left(\mathrm{c}^{2} \alpha+1\right) \mathrm{c} \frac{2 \pi}{n}+\mathrm{s}^{2} \alpha\right]$

由式(26)、(34)可知, 仅需一个运动变量 $\eta_{0,2}$ 即可完 全确定线对称条件下中间层单元的运动。

因为 $\kappa_{B 0,4}=0$ ，机构 $B_{\mathrm{s} 0}$ 的关节 4 固化，关节 3 和 5 合成一个关节, 退化为一个球面四杆机构。其 几何参数为 $\alpha_{12}^{B_{0}}=\alpha_{41}^{B_{0}}=\pi-\alpha-\beta, \alpha_{23}^{B_{0}}=\alpha_{34}^{B_{0}}=\beta$, 且其运动方程为

$$
\begin{gathered}
\tan \frac{\kappa_{B 0,2}}{2}=\frac{-\mathrm{s}(\alpha+\beta)+\sqrt{\mathrm{s}^{2}(\alpha+\beta)+\mathrm{s} \alpha \mathrm{s}(\alpha+2 \beta) \tan ^{2} \frac{\kappa_{B 0,3}+\kappa_{B 0,5}}{2}}}{\mathrm{~s} \alpha \tan \frac{\kappa_{B 0,3}+\kappa_{B 0,5}}{2}} \\
\tan \frac{\kappa_{B 0,1}}{2}=\frac{\mathrm{s} \beta \mathrm{s} \kappa_{B 0,2}}{\mathrm{~s}(\alpha+\beta) \mathrm{c} \beta+\mathrm{s} \beta \mathrm{c}(\alpha+\beta) \mathrm{c} \kappa_{B 0,2}} \\
\kappa_{B 0,6}=\kappa_{B 0,2} \quad \kappa_{B 0,3}=\eta_{0,6} \quad \kappa_{B 0,5}=\eta_{0,2}
\end{gathered}
$$

机构 $C_{\mathrm{s} 0}$ 仍为一个面对称球面六杆机构, 且其 运动方程同式(12c)。同理, 建立其他顶点的运动方 程, 可知其他层 Waterbomb 单元的运动仍保持面对 称特性, 且他们的运动方程如式(12d) (12g) 所示。

因此, 公式(26), (34), (35)和(12c) (12g)组成 了整个 Waterbomb 折纸管在扭转运动时的运动方程 组。从中可以看出, 仅需一个运动变量 $\eta_{0,2}$ 即可完 全确定整个管子的运动, 即该管子的自由度是 1 。 
以上 Waterbomb 折纸管的运动分析表明, 其中间层 的扭转运动是刚性的。下面我们将证明为何 Waterbomb 折纸管扭转运动的触发条件为管子中间 层单元到达完全折叠构型。也就是说, 当 $\kappa_{B 0,4} \neq 0$ 时, 球面机构 $A_{\mathrm{s} 0}$ 不会产生扭转运动。

图 11 给出了当 $\kappa_{B 0,4} \neq 0$ 时 Waterbomb 折纸管中 间层不完全折叠的情形，其坐标系的建立同图 10。 折痕 $B_{0}^{\prime} C_{-1}^{\prime}$ 与 $z$ 轴间的夹角 $\gamma$ 为

$$
\mathrm{c} \gamma=\frac{\tan \alpha}{a} \sqrt{\frac{-y_{B^{\prime} 0}^{2}+2 a y_{B^{\prime} 0} \mathrm{~s} \angle E A_{0} E^{\prime}-a^{2}}{\mathrm{c}^{2} \angle E A_{0} E^{\prime}}+\frac{a^{2}}{\mathrm{~s}^{2} \alpha}}
$$

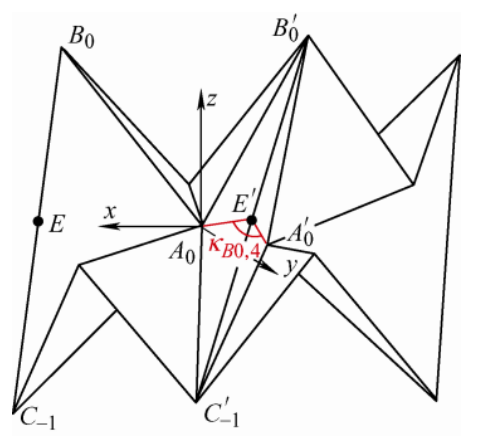

图 $11 \kappa_{B 0,4} \neq 0$ 时发生扭转的 Waterbomb 折纸管 中间层的两个相邻单元

由于面 $A_{0} E^{\prime} A_{0}^{\prime}$ 和面 $E A_{0} E$ 分别垂直于折痕 $B_{0}^{\prime} C_{-1}^{\prime}$ 与 $z$ 轴, 则两个面间的夹角也为 $\gamma$, 故顶点 $A_{0}^{\prime}$ 和 $A_{0}$ 间的坚直距离为

$$
z_{A^{\prime} 0}-z_{A 0}=-\overline{A_{0} A_{0}^{\prime}} \mathrm{s} \gamma=-2 a \mathrm{~s} \frac{\kappa_{B 0,4}}{2} \mathrm{~s} \gamma
$$

如果 $\kappa_{B 0,4} \neq 0$, 则 $z_{A^{\prime} 0}-z_{A 0} \neq 0$ 。根据公式(37) 中的递推公式可知, 随着中间层单元数目的增加, 顶点 $A_{0}^{\prime}$ 与面 $x A_{0} y$ 间的距离越来越大, 使得扭转后第 $n$ 个单元的顶点 $C_{-1}^{\prime}$ 与第 1 个单元的顶点 $C_{-1}$ 无法重 合, 故中间层单元无法形成一个周向闭环。因此, Waterbomb 折纸管能发生扭转运动的充要条件为中 间层单元达到完全折叠构型, 即 $\kappa_{B 0,4}=0$ 。下面, 我们将讨论不同几何参数条件下 Waterbomb 折纸管 刚性扭转运动的存在情况及对应的折叠角度范围。

Waterbomb 折纸管刚性扭转运动存在的条件为 其有一个完全折叠的线面对称层。这与几何参数 $\alpha$, $\beta, m$ 和 $n$ 均相关。当 $m$ 为奇数时, 其存在一个线 面对称的中间层, 所以我们需检查其是否存在完全 折叠的构型, 即需满足 $r_{A 0}=0$ 且 $r_{A i} \geq 0$ 。当 $n$ 给定 时, $\alpha$ 的范围由两个约束决定。当 $\alpha$ 增大时, 图 9 中的 $C_{0}^{\prime}$ 和 $B_{-1}$ 逐渐靠近。因此, 当折痕 $A_{0} C_{0}^{\prime}$ 与 $A_{0} B_{-1}$ 重合时, $\alpha$ 达到极限值, 如图 12 所示, 此时 $\angle E A_{0} C_{0}=\pi / n$ 。
由于 $\overline{C_{0} E}=\frac{a}{\mathrm{~s} \alpha \mathrm{s}(\alpha+\beta)} \sqrt{\mathrm{s}^{2} \alpha-\mathrm{c}^{2} \alpha \mathrm{s}^{2} \alpha+\beta}$ ， $\overline{A_{0} C_{0}}=\frac{a \mathrm{~s} \beta}{\mathrm{s} \alpha \mathrm{s}(\alpha+\beta)}, \overline{A_{0} E}=a$, 根据余弦定理可知

$$
\tan \alpha=1 / \mathrm{c} \frac{\pi}{n}
$$

式中， $\alpha$ 仅与 $n$ 相关。

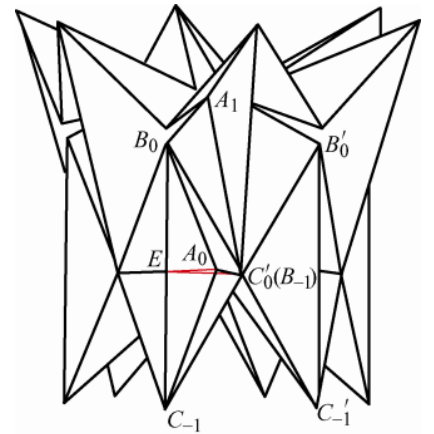

图 12 当 $A_{0} C_{0}^{\prime}$ 与 $A_{0} B_{-1}$ 重合时 $\alpha$ 达到极限值, 此时 $\beta$ 随机取为 $40^{\circ}$

$\alpha$ 的另一个约束可由当中间层完全折叠时第 $(m-1) / 2$ 层上的球面机构 $A_{\mathrm{s}[(m-1) / 2]}$ 完全展开获得。将 式(12)代入式(14)中, 可求得此时的 $\alpha$, 它不仅与 $n$ 有关, 还与 $\beta$ 和 $m$ 相关。 $\alpha$ 的最大值 $\alpha_{\text {max }}$ 是式(14)、 (38) 解的较小值。因此, 具有刚性扭转运动的 Waterbomb 折纸管 $\alpha$ 的取值范围为 $0<\alpha \leqslant \alpha_{\text {max }}$ 。

当 $\alpha$ 确定时, 中间层的相邻层 Waterbomb 单元 顶点 $A_{i}$ 的半径随着 $\beta$ 的增加而减小。当所有顶点 $A_{i}$ 的半径均为 0 , 即 $r_{A i}=0$ 时, $\beta$ 达到最大值。此时, 对于球面机构 $A_{\mathrm{s} 0}$

$$
\tan \frac{\eta_{0,2}}{2}=\operatorname{c} \alpha \tan \frac{\eta_{0,1}}{2} \quad \kappa_{B 0,4}=\eta_{0,1}-\frac{2 \pi}{n}=0
$$

对于球面机构 $B_{\mathrm{s} 0}$

$\tan \frac{\kappa_{B 0,2}}{2}=\frac{-\mathrm{s}(\alpha+\beta)+\sqrt{\mathrm{s}^{2}(\alpha+\beta)+\mathrm{s} \alpha \mathrm{s}(\alpha+2 \beta) \tan ^{2} \eta_{0,2}}}{\mathrm{~s} \alpha \tan \eta_{0,2}}$

对于球面机构 $C_{\mathrm{s} 0}$

$$
\tan \frac{\eta_{0,1}}{2}=\frac{2 \mathrm{~s} \beta \tan \frac{\kappa_{B 0,2}}{2}}{\mathrm{~s}(\alpha+2 \beta)+\mathrm{s} \alpha \tan ^{2} \frac{\kappa_{B 0,2}}{2}}
$$

因此, $\beta_{\text {max }}$ 可由以下公式求得

$\mathrm{c}^{4} \alpha \mathrm{s}^{2}(\alpha+\beta) \tan ^{4} \frac{\pi}{n}+2 \mathrm{c}^{2} \alpha\left[\mathrm{c} 2 \beta-\mathrm{c}^{2}(\alpha+\beta)\right] \tan ^{2} \frac{\pi}{n}+$

$\mathrm{s}^{2}(\alpha+\beta)-4 \mathrm{c}^{2} \alpha \mathrm{s}^{2} \beta=0$

此时, 所有层都处于线面对称的完全折叠状态, 这 意味着刚性扭转运动可以发生在任意层。当 
$\beta>\beta_{\text {max }}$ 时, $r_{A 1}<0,1$ 层发生干涉, 此时不存在刚 性扭转运动。当 $\beta<\beta_{\text {max }}$ 时, $r_{A 1}>0$, 刚性扭转运 动的存在性由第 $(m-1) / 2$ 层上的球面机构 $A_{\mathrm{s}[(m-1) / 2]}$ 的 完全展开构型决定, 即 $\eta_{(m-1) / 2,1}=\pi$ 。此时, $\beta$ 达到 最小值 $\beta_{\text {min }}$, 其值可由式(14)求得。 $\beta_{\text {min }}$ 与 $\alpha, m$ 和 $n$ 均相关。因此, 对于特定 $\alpha$ 的 Waterbomb 折纸 管, 其具有刚性扭转运动的 $\beta$ 的取值范围为 $\beta_{\text {min }} \leqslant \beta \leqslant \beta_{\text {max }}$ 。

当 $m$ 为偶数时, 除去 $r_{A i}=0$ 的情形, Waterbomb 折 纸管不存在线面对称的层, 故不会发生刚性扭转运动。

因此,一旦几何参数 $\alpha, \beta, m$ 和 $n$ 确定, 我 们即可根据以上分析确定刚性扭转运动的存在性。

下面我们将讨论刚性扭转运动存在时, 其折叠角度 $\eta_{0,2}$ 的取值范围。该取值范围由两个极限状态确定, 即 $\eta_{0,2}=0^{\circ}$ 或 $\eta_{0,3}=0^{\circ}$, 分别对应图 13 中的逆时针 扭转和顺时针扭转。

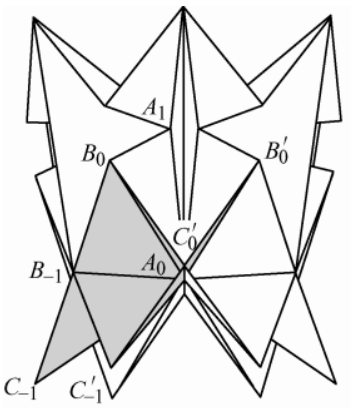

(a) $\eta_{0.2}=0^{\circ}$

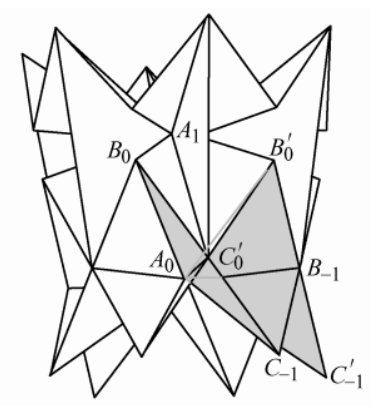

(b) $\eta_{0.3}=0^{\circ}$
图 13 扭转运动的两个极限状态

将 $\eta_{0,3}=0^{\circ}$ 代入式(34)中, 可求得图 $13 \mathrm{~b}$ 中 $\eta_{0,2}$ 的极限值为

$W_{1} \mathrm{c}^{4} \eta_{0,2}+W_{2} \mathrm{c}^{3} \eta_{0,2}+W_{3} \mathrm{c}^{2} \eta_{0,2}+W_{4} \mathrm{c} \eta_{0,2}+W_{5}=0$

其中, $W_{i}(i=1,2, \cdots, 5)$ 为 $\alpha$ 和 $n$ 的函数, 具体有

$$
\begin{gathered}
W_{1}=\mathrm{s}^{2} \alpha \mathrm{c}^{4} \alpha \\
W_{2}=2 \mathrm{~s}^{2} \alpha \mathrm{c}^{2} \alpha\left[\left(1-4 \mathrm{c}^{2} \alpha\right) \mathrm{c} \frac{2 \pi}{n}+1+2 \mathrm{c}^{2} \alpha\right] \\
W_{3}=\left(-16 \mathrm{c}^{6} \alpha+24 \mathrm{c}^{4} \alpha-9 \mathrm{c}^{2} \alpha+1\right) \mathrm{c}^{2} \frac{2 \pi}{n}+2\left(12 \mathrm{c}^{6} \alpha-\right. \\
\left.9 \mathrm{c}^{4} \alpha-4 \mathrm{c}^{2} \alpha+1\right) \mathrm{c} \frac{2 \pi}{n}-14 \mathrm{c}^{6} \alpha+12 \mathrm{c}^{4} \alpha+\mathrm{c}^{2} \alpha+1 \\
W_{4}=2\left[\mathrm{~s}^{2} \alpha\left(16 \mathrm{c}^{4} \alpha-1\right) \mathrm{c}^{2} \frac{2 \pi}{n}+\left(28 \mathrm{c}^{6} \alpha-31 \mathrm{c}^{4} \alpha+\right.\right. \\
\left.\left.5 \mathrm{c}^{2} \alpha-2\right) \mathrm{c} \frac{2 \pi}{n}-10 \mathrm{c}^{6} \alpha+7 \mathrm{c}^{4} \alpha+4 \mathrm{c}^{2} \alpha-1\right] \\
W_{5}=\left(-16 \mathrm{c}^{6} \alpha+8 \mathrm{c}^{4} \alpha+7 \mathrm{c}^{2} \alpha+1\right) \mathrm{c}^{2} \frac{2 \pi}{n}+2\left(20 \mathrm{c}^{6} \alpha-\right. \\
\left.19 \mathrm{c}^{4} \alpha-\mathrm{c} 2 \alpha\right) \mathrm{c} \frac{2 \pi}{n}-25 \mathrm{c}^{6} \alpha+35 \mathrm{c}^{4} \alpha-11 \mathrm{c}^{2} \alpha+1
\end{gathered}
$$

另一方面, 通过分析这个 Waterbomb 管子的运 动方程组, 即式(26), (34), (35)和(12c) (12g), 可
知其在发生扭转时同时张大。所以, $\eta_{0,2}$ 的另一个 极限由第 $(m-1) / 2$ 层上的球面机构 $A_{\mathrm{S}[(m-1) / 2]}$ 的完全展 开构型决定, 即 $\eta_{(m-1) / 2,1}=\pi$ 。通过求解公式(14), 可得到此时 $\eta_{0,2}$ 的解。将这些解与图 13 中的两个极 限值进行比较, 即可确定 Waterbomb 折纸管发生刚 性扭转运动时 $\eta_{0,2}$ 的取值范围。

需注意, 与刚性收缩运动类似, Waterbomb 折纸管除了扭转层外的其他层做面对称运动, 因 此在确定折叠角度的范围时需考虑面板间的干 涉。在运动过程中, 需始终满足条件 $r_{A i} \geqslant 0$ 和 $\kappa_{B i, 4} \geqslant 0$ 。通过求解运动方程组式(26)、(34)、(35) 和 $(12 \mathrm{c}) \sim(12 \mathrm{~g})$, 可进一步确定没有干涉的 $\eta_{0,2}$ 的 取值范围。

下面我们将讨论不同几何参数对 Waterbomb 折 纸管刚性扭转运动的影响。以 $n=6, \alpha=40^{\circ}$ 和 $\beta=40^{\circ}$ 的 Waterbomb 折纸管为例, 其刚性扭转运动 范围与层数 $m$ 间的关系如图 14a 所示, 其中 $m$ 为从 3 到 13 的奇数。由图 $14 \mathrm{a}$ 可以看出, 当 $m$ 增大时, $\eta_{0,2 \mathrm{~min}}$ 增大, 而 $\eta_{0,2 \text { max }}$ 减小, 则该管子刚性扭转运动范围递 减。当 $m \geqslant 13$ 时, 该管子第 6 层单元不满足周向闭 环条件, 其不再具有刚性扭转运动。

当 $m=5, \alpha=40^{\circ}, \beta=40^{\circ}$ 时, 为使 Waterbomb 折纸管具有刚性扭转运动, $n \geqslant 5$ 。图 $14 \mathrm{~b}$ 给出了此 时该管子刚性扭转运动范围与每层单元数 $n$ 间的关 系, 其中 $n$ 为从 5 到 20 的整数。由图 $14 \mathrm{~b}$ 可以看出, 当 $n$ 增大时, 该管子具有刚性扭转运动的 $\eta_{0,2 \min }$ 先减 小后保持不变, 而 $\eta_{0,2 \text { max }}$ 先增大后减小, 其刚性扭转 运动范围先增大后减小。

当 $n=6, m=5, \beta=40^{\circ}$ 时, 由式(38)可求得 具有刚性扭转运动的 Waterbomb 管子 $\alpha$ 取值范围为 $25.7^{\circ} \leqslant \alpha \leqslant 49.1^{\circ}, \alpha$ 与该管子刚性扭转运动范围 的关系如图 $14 \mathrm{c}$ 所示。由图 $14 \mathrm{c}$ 可以看出, 当 $25.7^{\circ} \leqslant \alpha<26.9^{\circ}$ 时, 该管子的刚性扭转运动范围递 增, 且管子的最顶层发生物理干涉, 如图中红线所示, 发生物理干涉的范围与 $\alpha$ 正相关。此时，若 Waterbomb 管子要进行扭转运动, 必然存在面内变 形, 该管子存在机构运动-结构变形相互转换的混合 模式。当 $\alpha \geqslant 26.9^{\circ}$ 时, 随着 $\alpha$ 的增大, $\eta_{0,2 \min }$ 先减 小后在 $\alpha \geqslant 35.7^{\circ}$ 时保持不变, 而 $\eta_{0,2 \max }$ 减小, 其刚 性扭转运动范围先增大后减小, 且发生物理干涉的范 围逐渐减少, 直至 $\alpha=37.3^{\circ}$ 时不再存在物理干涉。在 此过程中, Waterbomb 折纸管存在机构运动-结构变 形-机构运动相互转换的混合模式。 


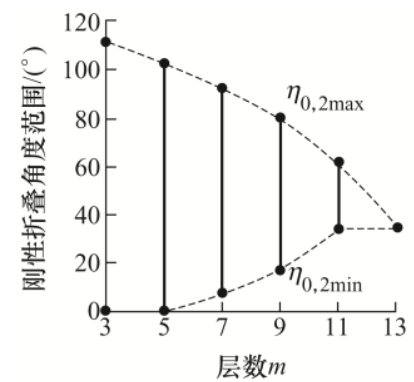

(a) 层数 $m$ 的影响

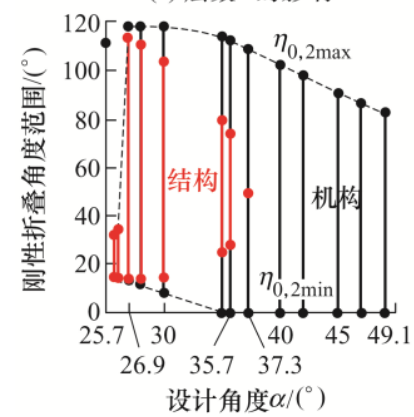

(c) 设计角度 $\alpha$ 的影响

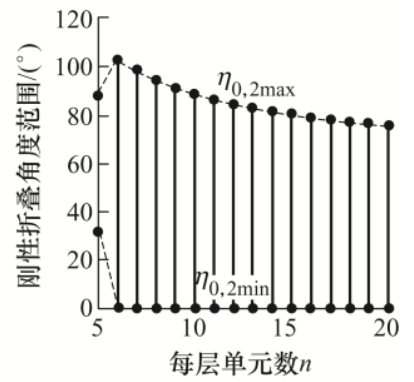

(b) 每层单元数 $n$ 的影响

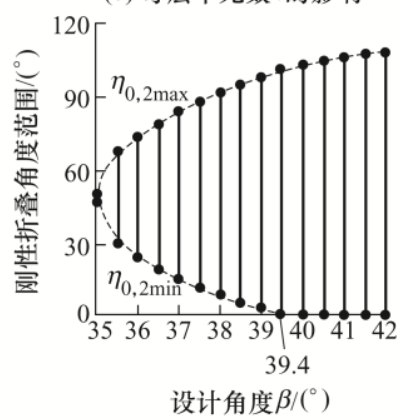

(d) 设计角度 $\beta$ 的影响
图 14 几何参数对 Waterbomb 折纸管扭转运动的影响

当 $n=6, m=5, \alpha=40^{\circ}$ 时, 由式(14)、(40) 可求得具有刚性扭转运动的 Waterbomb 折纸管的 $\beta$ 的取值范围为 $35^{\circ}<\beta \leqslant 42^{\circ}$, 且 $\beta$ 与管子刚性扭转 运动范围的关系如图 14d 所示。由图 14d 可以看出, 随着 $\beta$ 的增大, $\eta_{0,2 \min }$ 先减小后在 $\beta \geqslant 39.4^{\circ}$ 时保持 不变, 而 $\eta_{0,2 \text { max }}$ 增大, 且其刚性扭转运动范围递增。

另外, 刚性扭转运动仅发生在完全折叠的线面对 称层。一旦 Waterbomb 折纸管开始扭转, 完全折叠层 的相邻层张大, 其不满足扭转发生的触发条件, 故刚 性扭转运动不能在不发生面内变形的情况下传递到 其他层。从运动学角度看, 在整个刚性扭转运动过程 中, $\kappa_{B 0,4}=0$ 始终成立, 而 $\kappa_{C 0,4}$ 始终大于 0 。以 $m=5$, $n=6$ 的 Waterbomb 折纸管为例, 我们讨论 $\alpha_{1}=40^{\circ}$, $\alpha_{2}=45^{\circ}$ 和 $\alpha_{3}=50^{\circ}$ 这三种情形。通过求解公式(40), 可知三种情况对应的 $\beta$ 极限值分别为 $\beta_{1 \text { max }}=42.01^{\circ}$, $\beta_{2 \max }=45.59^{\circ}$ 和 $\beta_{3 \max }=49.17^{\circ}$, 此时 Waterbomb 折 纸管为所有层完全折叠的等直径圆柱。

取 $\beta=40^{\circ}$, 图 15a 给出了三种不同 $\alpha$ 的 Waterbomb 折纸管二面角 $\kappa_{B i, 4}, \kappa_{C i, 4}$ 与 $\eta_{0,2}$ 间的关系曲 线, 其中实线、虚线和点划线分别表示 $\alpha_{1}=40^{\circ}$, $\alpha_{2}=45^{\circ}$ 和 $\alpha_{3}=50^{\circ}$ 的情形。从中可以看出 $\kappa_{C i, 4}>0$, 即其他层不存在刚性扭转运动。同时, 在 $\beta$ 给定的情 况下, 刚性扭转运动的 $\eta_{0,2}$ 的取值范围随着 $\alpha$ 的增加 而减小。

保持 $\alpha, m$ 和 $n$ 的值不变, $\kappa_{C 0,4}$ 的最小值随着 $\beta$ 的增加而减小。图 $15 \mathrm{~b}$ 分别给出了三种不同 $\alpha$ 值下 $\beta$
取对应最大值的情况。此时, 当 Waterbomb 折纸管处 于等直径构型时, $\kappa_{C i, 4}=0$, 如图 $15 \mathrm{~b}$ 中的圆点所示。 在此构型下, 刚性扭转运动可以发生在任意层, 即该 构型是个分岔点。

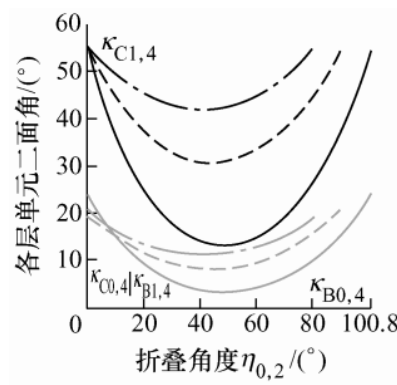

(a) $\beta$ 取相同值 $40^{\circ}$

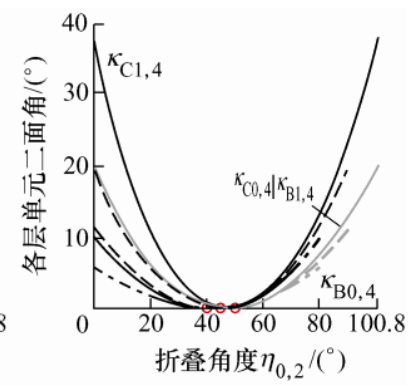

(b) $\beta$ 取对应最大值
图 15 三种不同 $\alpha$ 的 Waterbomb 折纸管的二面角 $\kappa_{B i, 4}$,

$$
\kappa_{C i, 4}(i=0,1) \text { 与 } \eta_{0,2} \text { 间的关系曲线 }
$$

因此, 当 $\alpha$ 和 $n$ 的值给定时, 公式(40)给出了具 有完全折叠的等直径构型的 Waterbomb 折纸管 $\beta$ 的 取值。在此情形下, 刚性扭转运动可以通过分岔点由 中间层转化到任意层。例如, 当 $m=3, n=6$ 且 $\alpha=40^{\circ}$ 时, 此分岔现象发生的条件为 $\beta=42.01^{\circ}$ 。

图 16 描述了此几何参数条件下的 Waterbomb 折纸管 的分岔运动及对应的 1 层二面角 $\kappa_{B 1,4}$ 与 $\eta_{0,2}$ 间的关系 曲线, 其中实线、虚线和点划线分别对应路径 I、路 径 II 和路径 III, 即分岔发生在最顶层(1 层)、中间层 (0 层)和最底层 $(-1$ 层)的情形。
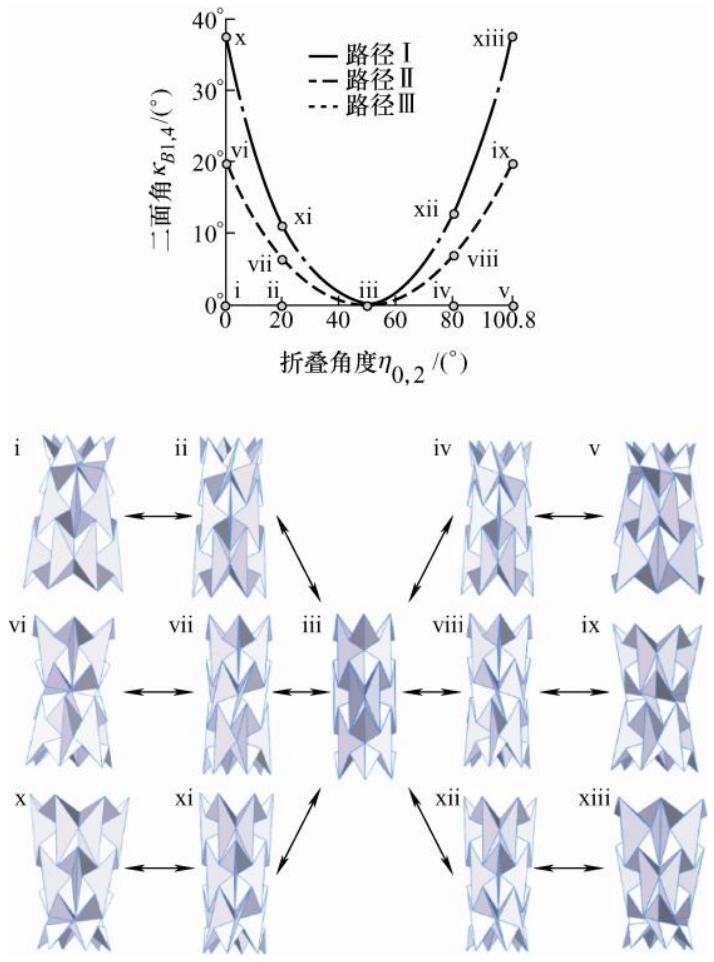

图 16 具有完全折叠等直径构型的 Waterbomb 折纸管的分岔 
如图 16 所示, 构型 $\mathrm{i}$, ii, iii, iv 和 v 对应路径 I 下刚性扭转运动发生在顶层的情形, 在构型 iii 的位 置, 它既可以分岔到路径 II(刚性扭转运动发生在中间 层, 运动构型分别为 vi, vii, iii, viii 和 ix), 也可以 分岔到路径 III(刚性扭转运动发生在最底层, 运动构 型分别为 $\mathrm{x}, \mathrm{xi}$, iii, xii 和 xiii)。构型 iii 对应 Waterbomb 折纸管的等直径完全折叠构型。

综上所述, Waterbomb 折纸管的刚性径向收缩 和扭转运动过程中各二面角间的关系如表 1 所示。

表 1 Waterbomb 折纸管刚性折叠运动的二面角

\begin{tabular}{|c|c|c|c|}
\hline \multirow{2}{*}{ 二面角 } & \multicolumn{2}{|c|}{ 径向收缩运动 } & \multirow{2}{*}{$\frac{\text { 扭转运动 }}{m \text { 只能为奇数 }}$} \\
\hline & $m$ 为奇数 & $m$ 为偶数 & \\
\hline$\eta_{0,1}$ & \multicolumn{2}{|c|}{ 输入 } & 公式(26a) \\
\hline$\eta_{0,2}$ & 式(12a) & $\kappa_{B 0,3}$ & 输入 \\
\hline$\eta_{0,3}$ & $\eta_{0,2}$ & 式(22b) & $\eta_{0,6}$ \\
\hline$\eta_{0,4}$ & $\eta_{0,1}$ & 式(22b) & $\eta_{0,1}$ \\
\hline$\eta_{0,5}$ & $\eta_{0,2}$ & $\eta_{0,3}$ & $\eta_{0,2}$ \\
\hline$\eta_{0,6}$ & $\eta_{0,2}$ & $\kappa_{B 0,3}$ & 式(34) \\
\hline$\kappa_{B 0,1}$ & 式(12b) & $\eta_{0,1}$ & 式(35) \\
\hline$\kappa_{B 0,2}$ & 式(12b) & 式(21) & 式(35) \\
\hline$\kappa_{B 0,3}$ & $\eta_{0,2}$ & 式(22a) & $\eta_{0,6}$ \\
\hline$\kappa_{B 0,4}$ & 式(12b) & 式(19) & 0 \\
\hline$\kappa_{B 0,5}$ & $\eta_{0,2}$ & $\kappa_{B 0,3}$ & $\eta_{0,2}$ \\
\hline$\kappa_{B 0,6}$ & \multicolumn{3}{|c|}{$\kappa_{B 0,2}$} \\
\hline$\kappa_{C 0,1}$ & \multicolumn{3}{|c|}{$\eta_{0,1}$} \\
\hline$\kappa_{C 0,2}=\kappa_{C 0,6}$ & \multicolumn{3}{|c|}{$\kappa_{B 0,2}$} \\
\hline$\kappa_{C 0,3}=\kappa_{C 0,5}$ & 式(12c) & $\kappa_{B 0,3}$ & 式(12c) \\
\hline$\kappa_{C 0,4}$ & 式(12c) & $\kappa_{B 0,4}$ & 式(12c) \\
\hline$\eta_{1,1}$ & 式(12d) & $\eta_{0,4}$ & 式(12d) \\
\hline$\eta_{1,2}=\eta_{1,6}$ & 式(12d) & $\eta_{0,5}$ & 式(12d) \\
\hline$\eta_{1,3}=\eta_{1,5}$ & $\kappa_{C 0,3}$ & $\eta_{0,6}$ & $\kappa_{C 0,3}$ \\
\hline$\eta_{1,4}$ & \multicolumn{3}{|c|}{$\kappa_{B 0,1}$} \\
\hline$\kappa_{B i, 1}$ & \multicolumn{3}{|c|}{ 式(12e) } \\
\hline$\kappa_{B i, 2}=\kappa_{B i, 6}$ & \multicolumn{3}{|c|}{ 式(12e) } \\
\hline$\kappa_{B i, 3}=\kappa_{B i, 5}$ & \multicolumn{3}{|c|}{$\eta_{i, 6}$} \\
\hline$\kappa_{B i, 4}$ & \multicolumn{3}{|c|}{$\kappa_{C(i-1), 4}$} \\
\hline$\kappa_{C i, 1}$ & \multicolumn{3}{|c|}{$\eta_{i, 1}$} \\
\hline$\kappa_{C i, 2}=\kappa_{C i, 6}$ & \multicolumn{3}{|c|}{$\kappa_{B i, 2}$} \\
\hline$\kappa_{C i, 3}=\kappa_{C i, 5}$ & \multicolumn{3}{|c|}{ 式(12f) } \\
\hline$\kappa_{C i, 4}$ & \multicolumn{3}{|c|}{ 式(12f) } \\
\hline$\eta_{i+1,1}$ & \multicolumn{3}{|c|}{ 式(12g) } \\
\hline$\eta_{i+1,2}=\eta_{i+1,6}$ & \multicolumn{3}{|c|}{ 式(12g) } \\
\hline$\eta_{i+1,3}=\eta_{i+1,5}$ & \multicolumn{3}{|c|}{$\kappa_{C i, 3}$} \\
\hline$\eta_{i+1,4}$ & \multicolumn{3}{|c|}{$\kappa_{B i, 1}$} \\
\hline
\end{tabular}

\section{4 结论}

（1）本文对一般几何参数条件下的广义 Waterbomb 折纸管的刚性折叠行为进行了完整的运 动学分析。采用刚性折纸与球面机构的等效原理并 基于 DH 矩阵法, 建立了奇数和偶数层 Waterbomb 折纸管所有二面角间的运动关系。结果表明, 在周 向和轴向对称的假设下, Waterbomb 折纸管可进行 刚性收缩运动且自由度为 1 。分析了刚性收缩运动 范围与管子几何参数间的关系, 不仅可由此分析给 定几何参数的 Waterbomb 折纸管的刚性运动范围, 也为设计具有刚性收缩运动的管子奠定了理论 基础。

（2）发现了某些 Waterbomb 折纸管具有刚性扭 转运动, 并给出了具体的几何参数条件。证明 Waterbomb 折纸管刚性折叠条件的触发条件是管子 中间层单元处于完全折叠构型。推导了刚性扭转运 动下 Waterbomb 管子的解析运动学方程, 并给出了 几何参数与刚性扭转运动范围的关系。

（3）讨论了对称假设条件下 Waterbomb 折纸管 的分岔行为。当该管子具有完全折叠的线面对称中 间层时, 其运动可由刚性收缩运动分岔到刚性扭转 运动。同时, 若该管子具有所有层完全折叠的等直 径构型, 其可实现各种不同层间刚性扭转运动的 转换。

本文的研究结果不仅有助于加深对 Waterbomb 折纸管运动学的理解, 还为其在超材料、可变形结 构和机器人中的应用提供了重要的理论支持, 同时 为复杂折纸结构的运动学分析提供了有效的手段。

\section{参 考 文 献}

[1] 杨名远, 马家耀, 李建民, 等. 基于厚板折纸理论的微 创手术钳[J]. 机械工程学报, 2018, 54(17): 36-45.

YANG Mingyuan, MA Jiayao, LI Jianmin, et al. Thick-panel origami inspired forceps for minimally invasive surgery[J]. Journal of Mechanical Engineering, 2018, 54(17): 36-45.

[2] WATANABE N, KAWAGUCHI K. The method for judging rigid foldability[J]. Origami, 2009, 4: 165-174.

[3] TACHI T. Generalization of rigid-foldable quadrilateralmesh origami[J]. Journal of the International Association for Shell and Spatial Structures, 2009, 50(3): 173-179.

[4] CAI J, ZHANG Y, XU Y, et al. The foldability of cylindrical foldable structures based on rigid origami[J]. 
Journal of Mechanical Design, 2016, 138(3): 031401.

[5] CAI J, LIU Y, MA R, et al. Nonrigidly foldability analysis of Kresling cylindrical origami[J]. Journal of Mechanisms and Robotics, 2017, 9(4): 041018.

[6] DAI J S, JONES J R. Mobility in metamorphic mechanisms of foldable/erectable kinds[J]. Journal of Mechanical Design, 1999, 121(3): 375-382.

[7] DAI J S, JONES J R. Kinematics and mobility analysis of carton folds in packing manipulation based on the mechanism equivalent[J]. Proceedings of the Institution of Mechanical Engineers, Part C: Journal of Mechanical Engineering Science, 2002, 216(10): 959-970.

[8] DAI J S, JONES J R. Matrix representation of topological changes in metamorphic mechanisms[J]. Journal of Mechanical Design, 2005, 127: 837-840.

[9] HULL T. Project origami : Activities for exploring mathematics[M]. CRC Press, 2012.

[10] WU W, YOU Z. Modelling rigid origami with quaternions and dual quaternions[J]. Proceedings of the Royal Society A: Mathematical, Physical and Engineering Sciences, 2010, 466(2119): 2155-2174.

[11] STREINU I, WHITELEY W. Single-vertex origami and spherical expansive motions[C]// Japanese Conference on Discrete and Computational Geometry. Springer, Berlin, Heidelberg, 2004: 161-173.

[12] WANG K, CHEN Y. Folding a patterned cylinder by rigid origami[J]. Origami, 2011， 5: 265-276.

[13] LIU S, LV W, CHEN Y, et al. Deployable prismatic structures with rigid origami patterns[J]. Journal of Mechanisms and Robotics, 2016, 8(3): 031002.

[14] CHEN Y, LÜ W, LI J, et al. An extended family of rigidly foldable origami tubes[J]. Journal of Mechanisms and Robotics, 2017, 9(2): 021002.

[15] LIU Y, YANG S. Kinematic solution of spherical Stephenson-III six-bar mechanism[J]. Chinese Journal of Mechanical Engineering, 2013， 26(5): 851-860.

[16] ZHUANG Y, ZHANG Y, DUAN X. Complete real solution of the five-orientation motion generation problem for a spherical four-bar linkage[J]. Chinese Journal of Mechanical Engineering, 2015, 28(2): 258-266.

[17] RANDLETT S. The art of origami: Paper folding, traditional and modern[M]. EP Dutton, 1961.

[18] ZHANG K, FANG Y, FANG H, et al. Geometry and constraint analysis of the three-spherical kinematic chain based parallel mechanism[J]. Journal of Mechanisms and
Robotics, 2010, 2(3): 031014.

[19] QIU C, ZHANG K, DAI J S. Repelling-screw based force analysis of origami mechanisms[J]. Journal of Mechanisms and Robotics, 2016, 8(3): 031001.

[20] KURIBAYASHI K, TSUCHIYA K, YOU Z, et al. Self-deployable origami stent grafts as a biomedical application of Ni-rich TiNi shape memory alloy foil[J]. Materials Science and Engineering: A, 2006, 419(1-2): 131-137.

[21] LEE D Y, KIM J S, KIM S R, et al. The deformable wheel robot using magic-ball origami structure[C]// ASME 2013 International Design Engineering Technical Conferences and Computers and Information in Engineering Conference. American Society of Mechanical Engineers Digital Collection, 2013.

[22] LEE D Y, KIM S R, KIM J S, et al. Origami wheel transformer: A variable-diameter wheel drive robot using an origami structure[J]. Soft Robotics, 2017, 4(2): 163-180.

[23] FANG H, ZHANG Y, WANG K W. An earthworm-like robot using origami-ball structures[C]// Active and Passive Smart Structures and Integrated Systems 2017. International Society for Optics and Photonics, 2017, 10164: 1016414.

[24] LI S, VOGT D M, RUS D, et al. Fluid-driven origami-inspired artificial muscles[J]. Proceedings of the National Academy of Sciences, 2017, 114(50) : 13132-13137.

[25] ONAL C D, WOOD R J, RUS D. An origami-inspired approach to worm robots[J]. IEEE/ASME Transactions on Mechatronics, 2012, 18(2): 430-438.

[26] FANG H, ZHANG Y, WANG K W. Origami-based earthworm-like locomotion robots[J]. Bioinspiration \& Biomimetics, 2017, 12(6): 065003.

[27] FENG H, MA J, CHEN Y, et al. Twist of tubular mechanical metamaterials based on Waterbomb origami[J]. Scientific Reports, 2018, 8(1): 1-13.

[28] MA J, FENG H, CHEN Y, et al. Folding of tubular waterbomb[J]. Research, 2020(1): 1735081.

[29] MUKHOPADHYAY T, MA J, FENG $\mathrm{H}$, et al. Programmable stiffness and shape modulation in origami materials: Emergence of a distant actuation feature[J]. Applied Materials Today, 2020, 19: 100537.

[30] 于靖军, 谢岩, 裴旭. 负泊松比超材料研究进展 [J]. 机 械工程学报，2018，54(13): 1-14. 
YU Jingjun, XIE Yan, PEI Xu. State-of-art of metamaterials with negative Poisson's ratio[J]. Chinese Journal of Mechanical Engineering, 2018, 54(13): 1-14.

[31] GILLMAN A, FUCHI K, BUSKOHL P R. Truss-based nonlinear mechanical analysis for origami structures exhibiting bifurcation and limit point instabilities[J]. International Journal of Solids and Structures, 2018, 147: 80-93.

[32] FONSECA L M, RODRIGUES G V, SAVI M A, et al. Nonlinear dynamics of an origami wheel with shape memory alloy actuators[J]. Chaos, Solitons \& Fractals, 2019, 122: 245-261.

[33] ZHAO Y, ENDO Y, KANAMORI Y, et al. Approximating 3D surfaces using generalized Waterbomb tessellations $[\mathrm{J}]$. Journal of Computational Design and Engineering, 2018, 5(4): 442-448.

[34] ZHAO Y, KANAMORI Y, MITANI J. Design and motion analysis of axisymmetric 3D origami with generic six-crease bases[J]. Computer Aided Geometric Design, 2018, 59: 86-97.
[35] WEI G , DAI J S. Origami-inspired integrated planar-spherical overconstrained mechanisms[J]. Journal of Mechanical Design, 2014, 136(5): 051003.

[36] DEMAINE E D, O'ROURKE J. Geometric folding algorithms: Linkages, origami, polyhedra[M]. Cambridge University Press, 2007.

[37] BEGGS J S. Advanced mechanism[M]. Macmillan, 1966.

[38] HARTENBERG R S，DENAVIT J. A kinematic notation for lower pair mechanisms based on matrices[J]. Journal of Applied Mechanics - Transactions of the ASME, 1955, 22: $215-221$.

作者简介: 冯慧娟, 女, 1990年出生, 博士。主要研究方向为可重构机 构、机构学、刚性折纸。

E-mail: huijuan@tju.edu.cn

马家耀, 男, 1986年出生, 博士, 副教授, 博士研究生导师。主要研究 方向为折纸结构、折展结构、薄壁吸能结构及微创手术医疗器械。

E-mail: jiayao.ma@tju.edu.cn

陈炎(通信作者), 女, 1974年出生, 博士, 教授, 博士研究生导师。主 要研究方向为可展结构、机构学、可重构机构、折叠结构等的基础理论 及其在航空航天结构、机器人、医疗微结构中的工程应用。

E-mail: yan_chen@tju.edu.cn 